\title{
Nanotechnology and nanocarrier-based approaches on treatment of degenerative diseases
}

\author{
Anindita Chowdhury $^{1}$ - Selvaraj Kunjiappan ${ }^{2}$ Theivendren Panneerselvam ${ }^{2}$. \\ Balasubramanian Somasundaram $^{2} \cdot$ Chiranjib Bhattacharjee $^{1}$
}

Received: 13 December 2016/Accepted: 24 April 2017/Published online: 28 April 2017

(c) The Author(s) 2017. This article is an open access publication

\begin{abstract}
Degenerative diseases are results of deterioration of cells and tissues with aging either by unhealthy lifestyle or normal senescence. The degenerative disease likely affects central nervous system and cardiovascular system to a great extent. Certain medications and therapies have emerged for the treatment of degenerative diseases, but in most cases bearing with poor solubility, lower bioavailability, drug resistance, and incapability to cross the bloodbrain barrier (BBB). Hence, it has to be overcome with conventional treatment system; in this connection, nanotechnology has gained a great deal of interest in recent years. Moreover, nanotechnology and nanocarrier-based approach drug delivery system could revolutionize the treatment of degenerative diseases by faster absorption of drug, targeted interaction at specific site, and its release in a controlled manner into human body with minimal side effects. The core objective of this review is to customize and formulate therapeutically active molecules with specific site of action and without affecting other organs and tissues to obtain effective result in the improvement of quality of health. In addition, the review provides a concise insight into the recent developments and applications of nanotech and nanocarrier-based drug delivery for the treatment of various degenerative diseases.
\end{abstract}

Selvaraj Kunjiappan

selvapharmabio@gmail.com

1 Department of Chemical Engineering, Jadavpur University, Kolkata 700032, West Bengal, India

2 Sir CV Raman-KS Krishnan International Research Center, Kalasalingam University, Krishnankoil 626126, Tamil Nadu, India
Keywords Degenerative diseases - Nanotechnology · Nanocarrier · Biocompatibility · Drug delivery $\cdot$ Drug resistance $\cdot$ Drug development $\cdot$ Bioavailability $\cdot$ Bloodbrain barrier

\section{Introduction}

Globally in the recent times there has been a deep change in food habit and lifestyle of people. The economically cheap junk foods, unhealthy eating habits, long work hours along with sedentary lifestyle have led to an inclination. It has resulted in epidemic of aging related to chronic degenerative/lifestyle/manmade diseases. The degenerative diseases are a group of heterogeneous disorder that is characterized by progressive degeneration of structure and function of system/organs. The report announced by World Health Organization (WHO) highlighted that among the countries India is one of the leading nations with most cases of degenerative diseases [1]. The major human degenerative diseases include Alzheimer's disease, Parkinson's disease, neoplastic diseases and cardiovascular diseases such as cardiopathies, coronary disease, myocardial infarction, hypertension, and cerebrovascular accidents/strokes. All of the above degenerative diseases are primarily associated with aging and these diseases affect millions of people around the world. In addition, in recent days, most of the countries were affected by major causes of mortality with certain types of degenerative diseases.

'Aging' or more specifically cellular aging and degenerative diseases exhibit cumulative results of increased levels of reactive oxygen species (ROS) due to oxidative stress [2]. In 1940s, theories of aging were stating that it was caused by mitochondrial dysfunction. Because of the importance of mitochondria and it plays a major role in 
generation of chemical energy, adenosine triphosphate (ATP), aging has an inverse correlation with production of ATP. Aged mammalian cells consist of higher amount of oxidized lipids and proteins along with damaged and mutated DNA in mitochondrial genome. As a result, gradually the mitochondria lose its ability to generate more energy and accumulate ROS. ROS are particularly active in the brain and neuronal tissue because metabolism of the excitatory amino acids and neurotransmitters generates huge amount of ROS. Thus, brain serves as a source of generation of oxidative stress [3]. ROS irruption in postmitotic cells such as glial cells and neurons leads to neuronal damage as they are particularly sensitive to free radicals. This in turn results in oxidative injury on human cells and in the end may lead to programmed cell death, i.e., apoptosis. These mechanisms eventually lead to neurodegenerative diseases and cancers [4].

Till date, no treatment has been found to be absolutely effective towards cancer. Among the vast number of therapies, chemotherapy is extensively used all over the world. Chemotherapy causes inconvenience for the patient because of its horrifying side effects. Therapeutically strong drugs are destroying the rapidly multiplying cells. Chemotherapy not only kills the malignant cells but adjacent healthy cells as well, inducing several side effects. The side effects caused by chemotherapy are so oppressive that patients often do not opt for such ailments. Due to nontargeted distribution throughout the body tissues, chemotherapeutic drugs which are administered as 'free drug' lead to the limitation of drug concentration at impaired organ sites. Such obstacles could be closely associated with the clinical failure of this therapeutic modality in degenerative diseases. Site-specific targeted drug delivery offers a potential alternative strategy for chemotherapy. Nanotechnology involves research with an amalgamation of chemistry, physics, engineering, biology, and medicine, and acts as a significant technique in the treatment of degenerative diseases, such as early detection of tumors, site-specific action; reduce multidrug resistance and toxicity, discovery of cancer biomarkers, and development of novel treatments [5]. Nanocarriers have been established to successfully deliver active drug molecules to the target site/cells [6]. Moreover, nanotechnology and nanocarrier-based drug delivery system offers improved healing efficacy and reduces undesirable side effects allied with conventional drugs, introduces new classes of therapeutics and persuades the re-investigation of pharmaceutically suboptimal but biologically active new molecular entity that were earlier considered undevelopable.

Nanoparticles used during drug delivery system are vehicles with smallest functional organization normally $<100 \mathrm{~nm}$ in at least one dimension, and are made of diverse biodegradable materials such as natural or synthetic polymers, lipids, or metals [7]. Nanotechnology and nanocarrier-based therapeutics have accelerated the development in the field of biomedicine when compared with traditional drugs, in terms of improved half-lives, retention, and targeting efficiency, and lesser adverse effects. Chemotherapeutic nanomedicines have brought a revolutionary change and several compounds are going through various stages of experimental trial or already accepted by U.S. Food and drug administration (FDA) [8]. With the growing global commercialization efforts, the rush in nanomedicine research during the past few decades now translates itself into commercial aspects, with many products on the market and growing number in the pipeline. Presently, nanomedicine is led by drug delivery systems, which account for additional $75 \%$ of total sales. Pharmaceutical industries are gaining increasing interest in wide spectrum of nanotechnological advances because these developments have changed the scientific landscape providing several advantages, such as customized release systems and the potential to fabricate new formulated device that were previously not possible (due to several aspects related to the active constituents). The first evidence of nanoparticle-delivered clinical RNA interference (RNAi) has been introduced by Calando Pharmaceuticals. However, BIND Biosciences has shown a significant reduction of lung and tonsillar lesions using the combination of nanoparticles and chemotherapeutic drug with prostate-specific membrane antigen (PSMA) with greater efficacy compared with the drug alone, and at substantially lower doses. Again, Celgene's Abraxane, Paclitaxel modified with albumin-functionalized formulation was initially approved by the FDA for therapy of breast cancer, but recently also gained approval for lung and pancreatic cancer therapies [9].

To increase the potency of treatment and lessen side effects, nanoengineered devices are fabricated. These include nanomedicine, such as carbon, inorganic nanoparticles, protein-based, lipid-based nanoparticles, polymer-based nanoparticles and conjugates, dendrimers, micelles, nanocrystals, fullerene, nanodevices, nanobots, and biological nanoparticles. A generalized overview of some of these new nanovehicles for drug release which aim to progress the bioavailability, pharmacokinetics, and pharmacodynamics of drugs are summarized in Table 1.

The purview of the review article is to highlight how these obstacles can be overcome by nanotechnology and nanocarrier-based approaches in drug delivery systems on degenerative diseases, the molecular mechanisms of the fundamental interactions of nanoparticles with cell-surface receptors, biological responses and cell signaling, and the research needed for the extensive application of nanodelivery systems in medicine. 







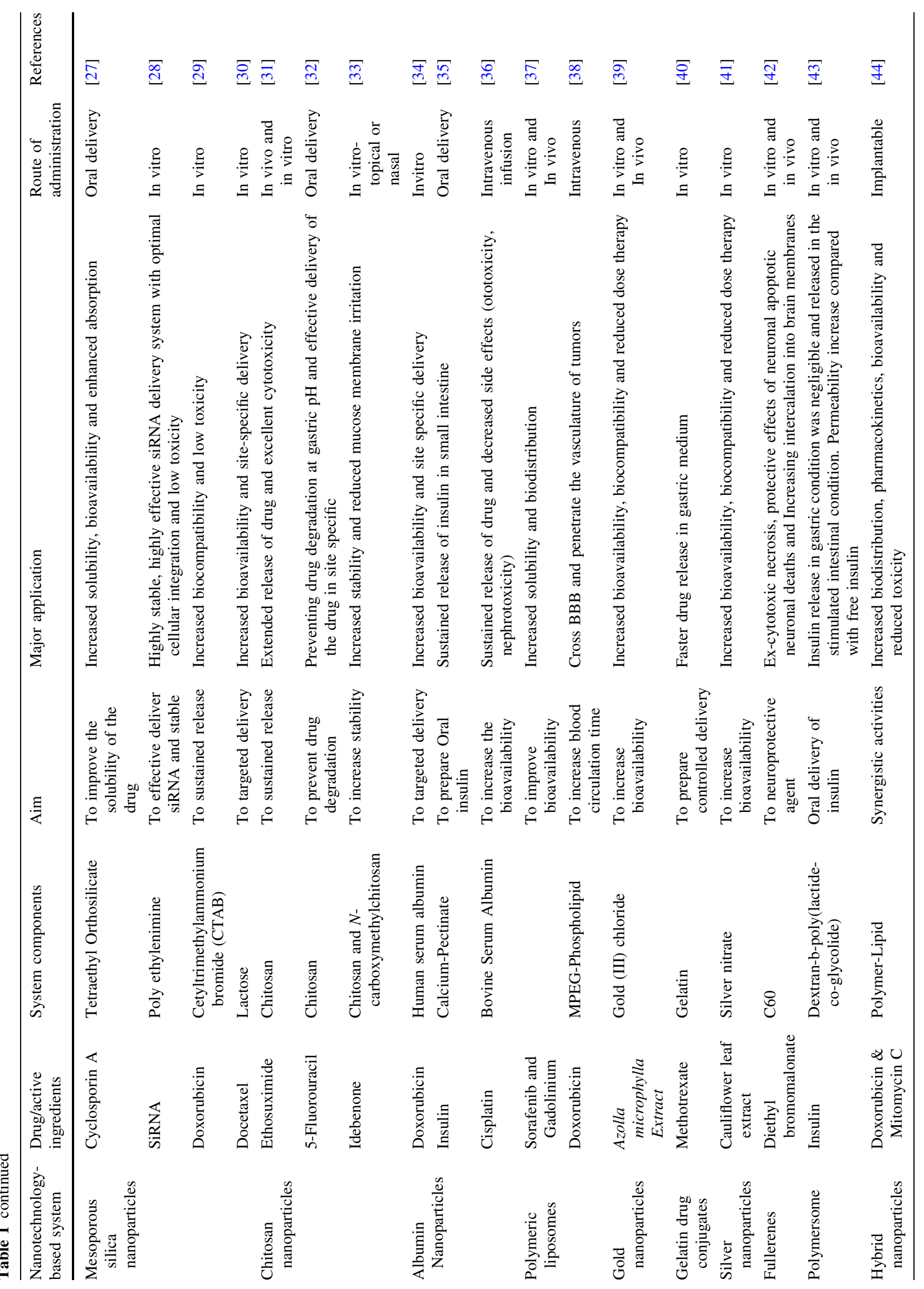




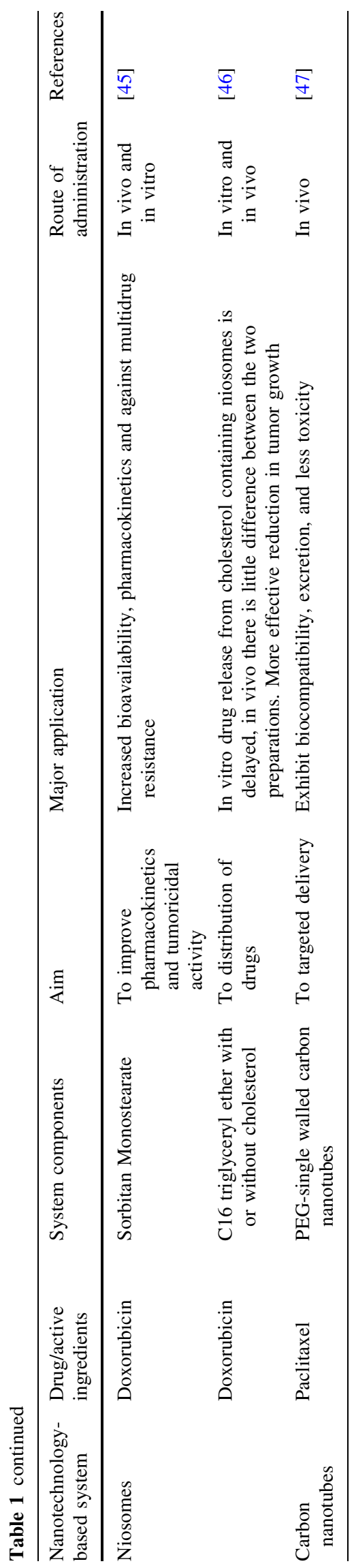
Design of nanotechnology and nanocarrier-based
drug delivery systems

The application of nanotechnology and nanocarrier for drug delivery systems has evolved with revolutionary effect in the biomedical landscape in recent years. Several research institutes and industries are conducting research programs extensively all over the world to find new formulations with specification capable of delivering drugs. Nanotechnology and nanocarrier-based chronic degenerative disease therapeutics have provided the possibilities of delivering drugs to specific cells using nanoparticles. Nanoscale complexes presently being developed consist of two main components: the nanovehicle, which is used as the carrier agent, and the chemotherapeutic drug [48]. The drug is usually confined within a membrane or a matrix system and can also be adsorbed, dissolved, or dispersed from the system [49]. Nanoparticles can be used to provide targeted delivery of drug at the specific site and thus enhance the uptake of poorly soluble drugs and bioavailability. A schematic evaluation of conventional and nanotechnology-based drug delivery systems is shown in Fig. 1. Nano-enabled structures are adapted to defend drugs from hydrolytic and enzymatic degradation. They also check drugs from first-pass metabolism and increase the blood residence time. The nano size allows them to penetrate through the tissues efficiently and may also pass biological barriers $[50,51]$. For instance, polymeric nanoparticles are encapsulated with Tacrine for intravenous drug delivery system to provide high concentrations of Tacrine in the brain and reduce the total dose required for the therapy [52]. Rivastigmine polymeric nanoparticle intravenous drug delivery systems offer high concentrations of Rivastigmine in brain [53]. Curcumin-phospholipid conjugate-based ex vivo liposomes delivery system provides strongly labeled $A \beta$ deposits [54]. Nanoparticles offer great visions of improved, personalized medicine which has emerged in recent days to enlarge and administer the suitable drug, at suitable dose, at the suitable time to the suitable patient. Advances in materials science and protein engineering have given rise to novel nanoscale targeting approaches that may increase safety and therapeutic efficacy in chronic degenerative diseases patients. The advantages of this approach depend on two aspects, size and biodegradable material, which in turn gave rise to better treatment modalities with accuracy, efficacy, safety, and speed.

\section{Types of nanotechnology and nanocarrier-based therapeutics used to treat degenerative diseases and recent developments in clinical status}

Nanotechnology and nanocarrier-based drug delivery systems are generally used to develop the effectiveness of therapeutic agents; the most common nanosystems used to 
Fig. 1 Design of nanotechnology-based drugs

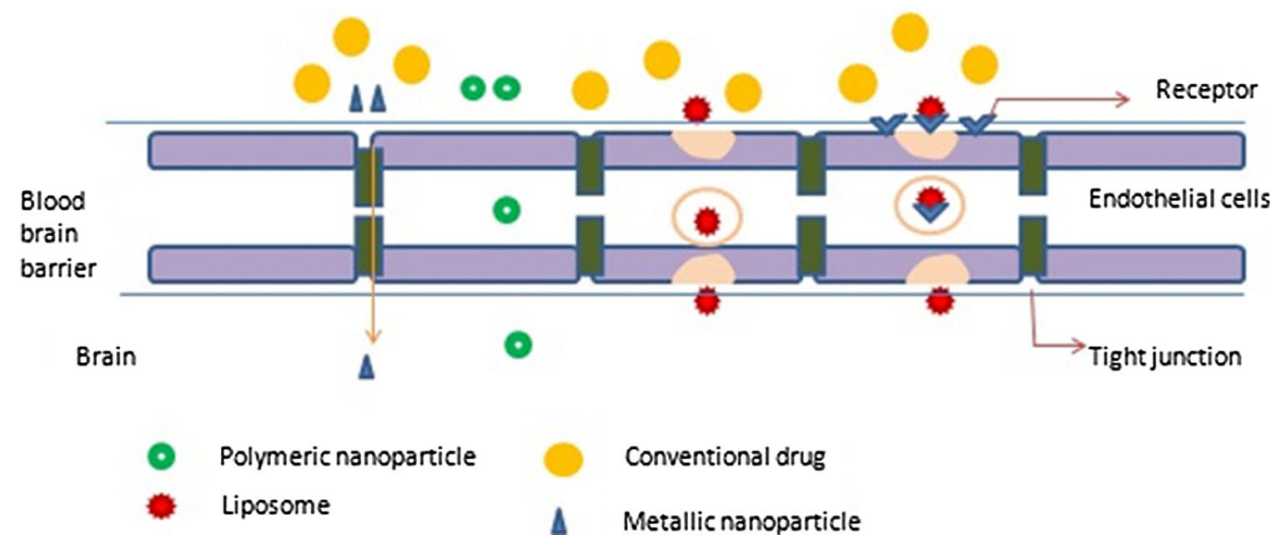

treat degenerative diseases are liposomes, polymeric nanoparticles, polymeric micelles, protein nanoparticles, inorganic nanoparticles, carbon nanotubes, polymeric conjugates, hybrid nanoparticles, solid lipid nanoparticles, niosomes, and dendrimers, which have been used to carry wide classes of therapeutic agents with cytotoxic agents, chemosensitizers, small interference RNA (siRNA), and antiangiogenic agents.

\section{Inorganic nanoparticles}

Over the years, inorganic nanoparticles have fascinated considerable interest due to their intriguing physicochemical properties, undersized and surface plasmon behavior [55]. Inorganic nanoparticles such as magnetic nanoparticles (iron oxide), gold, platinum, chromium, manganese, zinc, selenium, titanium, molybdenum, palladium, silica, copper, cerium oxide, and silver nanoparticles, bimetallic, nanoshells, and nanocages have been continuously used and modified to enable their use as a therapeutic and diagnostic agent. The existence of inorganic nanoparticles in solution was first recognized by Michael Faraday in 1857 and a quantitative explanation of their color was given by Mie in 1908 [56]. An inorganic nanoparticle exhibits a range of applications from catalysis and sense to optics, antibacterial activity, cytotoxic effects and data storage depending on size and shape [57]. For instance, the antibacterial activity and cytotoxicity effects of inorganic nanoparticles were closely related to their size as well as shape, that is, minor the metal nuclei, higher the activity [58]. The size of inorganic nanoparticles is mainly dependent on the metal salt concentration, temperature, rate of chemical reactants of the reaction medium [59]. Thus, control over the size and size allotment is the prime factor of concern.

Generally, specific shape, size, and size allotment is customized by modification of the synthesis methods, reducing agents and stabilizers [60-64]. Many processes are followed for the synthesis of nanoparticles. These include physical, chemical, and biological routes [65-67]. Physical approach utilizes numerous methods such as evaporation/condensation and laser ablation, whereas chemical approach involves the metal ions in solution which are reduced in conditions favoring the successive formation of small metal clusters or aggregates [68-70]. The physical and chemical methods suffer from several drawbacks such as high cost, use of toxic chemicals, demand for expensive instrument, more energy requirement, pressure and are not eco-friendly [71]. On the other hand, biologically mediated synthesis of nanoparticles is gaining significance due to its simplicity, single step, nontoxicity, biocompatible eco-friendliness and their unique physiochemical properties [72-74]. Nanoscale materials and supramolecular structures usually with a collection of shapes (spheres, rods, prisms, plates, needles, leafs or dendrites) and sub-micrometer sizes ranging from 1 to $100 \mathrm{~nm}(\mathrm{~nm})$ is an emerging area of nanoscience and nanotechnology. Here we depicted some important metallic nanoparticles used for non-infective disease drug delivery systems.

\section{Gold nanoparticles}

Gold is one of the most important and widely used noble metals due to its broad spectrum applications in industry and economic activity. It is the most attractive microelement that plays significant role in the field of bio-nanotechnology [75]. In the past 50 years, pure gold has been used as a food additive. WHO also recommended gold as a food additive in 1983 [76]. Colloidal gold has been used as medicinal agent for treating rheumatoid arthritis, alcoholism, tuberculosis and neoplastic disorders [77]. Currently, metal nanoparticles, especially gold nanoparticles (AuNPs) have drawn the attention of scientists because of their stability, oxidation resistance and biocompatibility. AuNPs provide many advantageous attributes for the creation of drug delivery systems. First, the core materials of gold are chemically static and non-cytotoxic [78]. Second, 
the unique nanosize dimension of AuNPs provides a large surface area readily available for modification with loading targeting molecules or specific biomarkers for drug delivery systems [79]. It can be utilize as a potential vehicle to deliver micromolecules such as proteins, DNA, or RNA [80]. It also enables ease of drug attachment through ionic or covalent bonds, or through adhesion. Like for many nanodrugs, PEG can be used as an attachment biomaterial on the surface of metallic nanoparticles to increase stability and circulation time, in addition to other targeting agents [81]. It has been investigated that AuNPs with diameter $\leq 50 \mathrm{~nm}$ can cross the BBB. Moreover, PEGylated AuNPs conjugated with TNF (tumor necrosis factor) can extravasate through tumor cells due to their leaky vasculature [82]. Several types of AuNPs have been extensively studied for siRNA delivery. These incorporate AuNPs functionalized with cationic quaternary ammonium or branched PEI, cationic lipid bilayer-coated AuNPS and oligonucleotide-modified AuNPS. Gold nanorods are also found to contain immense potential to deliver siRNA to target cells or tissues [83]. Gold nanorod-DARPP-32 siRNA complexes (nanoplexes) have been developed by group of scientists which can target and diminish expression of the key proteins (DARPP-32), extracellular signal-regulated kinase (ERK), and protein phosphatase 1 (PP-1) in the dopaminergic signaling pathway in the brain for ailment of drug addiction [84].

\section{Silver nanoparticles}

Silver nanoparticles (AgNPs) play a pivotal role in the field of chemistry (catalysis), physics (optical, electrical, and photothermal properties), and healthcare (therapeutics, diagnosis, and immunoassay) [85-88]. Several researches have conceded that AgNPs are widely known for its antimicrobial and anticancer activity [89]. The use of silver in nanoparticle form has reduced cellular toxicity but not antibacterial efficacy as compared to its ionic form. Certainly, the superior antibacterial properties of AgNPs contribute to the formation of free radicals from the surface of $\mathrm{Ag}$ [90]. Apart from being an excellent antibacterial agent, AgNPs appears to have anti-inflammatory properties as well. AgNPs reduced the production of pro-inflammatory cytokines such as interleukin-6 (IL-6), tumor necrosis factor-alpha (TNF- $\alpha$ ), and interferon-gamma (IFN- $\gamma$ ), although the intracellular pathways involved still remains largely not elucidated [91]. AgNPs are a popular choice in disease management because of their specific interaction with and disruption of the mitochondrial respiratory chain. AgNPs disrupt mitochondrial function by inducing the generation of ROS and suppressing ATP synthesis, which lead to DNA damage [92]. AgNPs have been proven to slow the progression of macular degeneration and other optical diseases. The AgNPs inhibit pathway of the growth factor that increases the permeability of endothelial cells. By inhibiting this permeability, AgNPs consequently slow the progression of optical degenerative diseases and can even increase visual acuity in some patients [93].

\section{Magnetic nanoparticles}

The conception of understanding magnetic micro- and nanoparticles and its application for drug delivery system was nourished in the late 1970s by Widder, Senyi and Colleagues [94, 95]. Such particles with superparamagnetism at room temperature are usually selected for biomedical applications. Owing to its high field irreversibility, high saturation field, superparamagnetism, and extra anisotropy, magnetic nanoparticles cover a broad spectrum of biomedical applications. These traits are due to the narrow and finite size effects and surface effects that define the magnetic behavior of individual nanoparticles [96]. Magnetic nanoparticles enable the systematic administration of drug to an exact target site in the human body while remaining eventually localized, due to applied magnetic field. The basic idea is that therapeutic agents are either attached to, or encapsulated in, magnetic microor nanoparticles. The polymers or metal/nonmetal coating acts as a support for the delivery of therapeutic drugs or nucleic acid. These particles contain magnetic cores encapsulated by polymer or metal coating, or may consist of porous polymers containing magnetic nanoparticle precipitate within the pores. Cytotoxic drugs or therapeutic DNA for targeted chemotherapy is attached by functionalizing the polymer or metal coating to correct a genetic defect. Magnetic nanoparticle technology thus offers the potential for selective and competent delivery of therapeutic genes or drugs due to external magnetic fields [97].

\section{Mesoporous silica nanoparticles}

Nanosized mesoporous silica particles (MSNs) with high chemical and thermal stability are excellent theranostic agents in drug delivery systems for targeted degenerative diseases as well as bioimaging devices. In comparison to the porous silica, MSNs are better candidates in drug delivery systems owing to its unique characteristics for their planned arrays of 2D hexagonal micro- or mesopore structure, uniform particle sizes $(80-500 \mathrm{~nm})$, high pore volumes $\left(0.5-2.5 \mathrm{~cm}^{3} / \mathrm{g}\right)$, large surface areas $\left(>1000 \mathrm{~m}^{2} /\right.$ $\mathrm{g}$ ), flexible pore diameters $(1.3-30 \mathrm{~nm})$, tunable particle morphology and both exterior and interior surfaces that could be autonomously modified with a variety of functional groups [98]. A high density of silanol groups 
dominates the silica surface which can be functionalized with a wide variety of organic functional groups [99]. This adaptation allows for targeting biomolecules such as peptides, antibodies or folic acid, or biocompatible polymers to minimize opsonization for rapid clearance of nanoparticles [100]. These flexible surface characteristics of MSNs have high drug-loading capacity. The MSNs are especially useful to mass therapeutic compounds such as enzymes that are easily degraded in the unfriendly biological environment when delivered without encapsulation. These therapeutic agents are either covalently attached, or adsorbed onto such silica nanocarriers that have been presurface modified. These approaches can easily defeat poor drug solubility and stability issues, besides having better control over the rate of drugs release [101].

\section{Polymer-based nanoparticles}

Polymeric nanoparticles are submicron colloidal solid particles ranging approximately from 10 to $1000 \mathrm{~nm}$ is increasingly gaining popularity owing to their better stability and higher encapsulation efficiency for delivery of drug to tumor cells. The drug is either loaded inside polymer or conjugated on the surface of polymeric nanoparticles. The polymeric coating enhances solubility of the hydrophobic drugs, provides stability from extracellular environment and lowers the toxicity of drug with high therapeutic ratio. These particles also permit controlled and persistent release of drug to the specific target sites.

They can target delivery of drug specifically to Central Nervous System (CNS) as their surface can be functionalized with other molecules to escape from recognition by macrophages present in reticuloendothelial system. The drugs designed for the treatment of neurodegenerative diseases are mostly lipophilic and have small molecular masses so that they can easily penetrate through the BBB [102]. Till now the gold standard for the treatment of neurodegenerative diseases such as Parkinson's disease is the oral administration of dopamine antagonist levodopa but the efficacy of levodopa diminishes rapidly [103]. In this context nanotechnology has achieved targeting delivery of dopamine in brain. It has been revealed by in vitro test that dopamine-loaded chitosan nanoparticles have reduced the cytotoxicity of free dopamine [104]. Levodopa nanoparticle-encapsulated benserazide poly(lactic-co-glycolic acid) (PLGA) has successfully removed duskiness in rat. It has been also used for preparing thermoreversible gel using Pluronic PF127 and found to increase the drug levels in brain with better efficacy [105].

One of the polymers found to be highly effective as a coating material for nanoparticle in degenerative diseases is polyethylene glycol (PEG). It is either used for encapsulation or surface modification owing to its targeting capabilities and avoiding uptake by reticuloendothelial system. Studies performed on mice with tumor of MDAMB231 has shown an extended circulation time and enhanced tumor targeting with polyethylene oxide-modified polyepsilon-caprolactone [106]. Biodegradable polymers such as chitosan and collagen or non-biodegradable polymers such as polyvinyl alcohol (PVA), PEG, monomethoxy poly-(ethylene glycol) (mPEG), polysorbate are some of the blood compatible polymers which have been used for the development.

\section{Niosomes}

Niosomes are nanometric-scale class of vesicular drug delivery systems with a bilayer membrane as well as hollow space. In niosomes, medication is encapsulated in a vesicle and it is composed of bilayer-hydrated non-ionic surface active agents such as cholesterol or its derivatives and hence the name niosomes [107]. This unique structure of niosomes allows encapsulation of both hydrophilic and lipophilic substances. This can be achieved by entrapment of hydrophilic substances in vesicular aqueous core or adsorption on the bilayer surfaces while the lipophilic substances are encapsulated by their partitioning into the lipophilic domain of the bilayers. The vesicles are categorized by a higher chemical stability with respect to liposomes due to the variation in composition between surfactants and phospholipids but appear to be similar in terms of the physical properties of liposomes being prepared in the same way [108]. The chemical stability as well as cost effectiveness of the materials for the synthesis of niosomes made these vesicles more attractive than liposomes as potential drug delivery system. Niosomes have been reported to decline side effects, give sustain release and to enhance penetration of the trapped drug through intravenous, intramuscular, oral, ocular, pulmonary and subcutaneous routes. Vesicles act as penetration enhancer reducing the obstacle in stratum corneum, which will result in reduction of trans-epidermal water loss and thereby increasing the smoothness via replenishing lost skin lipids [109]. In addition, niosomes have been shown to develop absorption of some drugs across cell membranes, and localize in targeted organs and tissues to elude the reticuloendothelial system [110].

\section{Polymer-drug conjugates nanoparticles}

Polymer-drug conjugates are gaining importance as major types of nanocarriers and are currently in clinical trials as advanced as phase III. This polymer-drug conjugates system entraps drug molecules in polymer molecules. 
Polymer-drug conjugates are synthesised by side-chain grafting of drugs to polymer chains, which allows the delivery of high doses of chemotherapeutic drugs [111]. The existence of the polymer increases the solubility of the hydrophobic drug and improves its pharmacokinetic profile; on the one hand, it increases plasma half-life along with volume of distribution and on the other, it reduces clearance by the kidneys or liver. The polymer also protects the drug against degradation [112]. Polymer-drug conjugate has three major components, a soluble polymer backbone, a biodegradable linker, and covalently linked anticancer drug which is deactivated as a conjugate. The polymer drug linker is cleaved to release and re-activate the attached drug molecules [113]. Despite the variety of novel drug targets and complicated chemistries available, only four drugs (doxorubicin, camptothecin, paclitaxel, and palatinate) and four polymers ( $N$-(2-hydroxypropyl) methacrylamide (HPMA) copolymer, poly-L-glutamic acid, PEG, and dextran) have been frequently used to develop polymer-drug conjugates.

\section{Polymersome nanoparticles}

Polymersomes (Ps) (also referred to as polymeric vesicles) or polymer-based colloidal carriers have attracted rapidly growing interest based on their stimulating aggregation phenomena, cell and virus-mimicking dimensions and functions [114]. Ps are artificial vesicles that contain an aqueous solution in the core bounded by a bilayer membrane. The bilayer membrane is composed of hydrated hydrophilic coronas (e.g., PEG) together inside and outside of hydrophobic middle part of the membrane. The aqueous core can be utilized for the encapsulation of therapeutic hydrophilic molecules and the membrane can amalgamate hydrophobic drugs within its hydrophobic part [115-117]. Due to the relatively thick membranes, Ps can be rather stable. The existence of a hydrophilic PEG brush on the surface will reduce the protein adsorption onto the Ps during the blood circulation. Permeability, rate of degradation and stimuli-sensitivity of the membranes can be varied using various biodegradable and/or stimuli-responsive block copolymers to modulate the release of the encapsulated drugs. End groups of the PEG can be utilize to immobilize homing moieties like antibodies or arginineglycine-aspartic acid (RGD)-containing peptides, which are able to recognize target cells or tissues.

\section{Dendrimers}

Dendrimers are globular polymeric macromolecular threedimensional structures, which are hyperbranched and well organized as shown in Fig. 2. The nanoscopic size, narrow polydispersity index, outstanding control over molecular structure, presence of multiple functional groups at the margin and cavities in the interior are some of the unique characteristics associated with these dendrimers, which distinguish them amongst the available polymers and have displayed a crucial role in the emerging field of nanomedicine [118]. The name dendrimer derived from the Greek word "Dendron", which means "tree", indicates their unique tree-like branching architecture. They are characterized by layers between each cascade point popularly known as "Generations". The complete architecture of dendrimer can be classified into the inner core moiety
Fig. 2 A diagrammatic explanation of different classes of nanotechnology and nanocarrier-based drug delivery systems for degenerative diseases

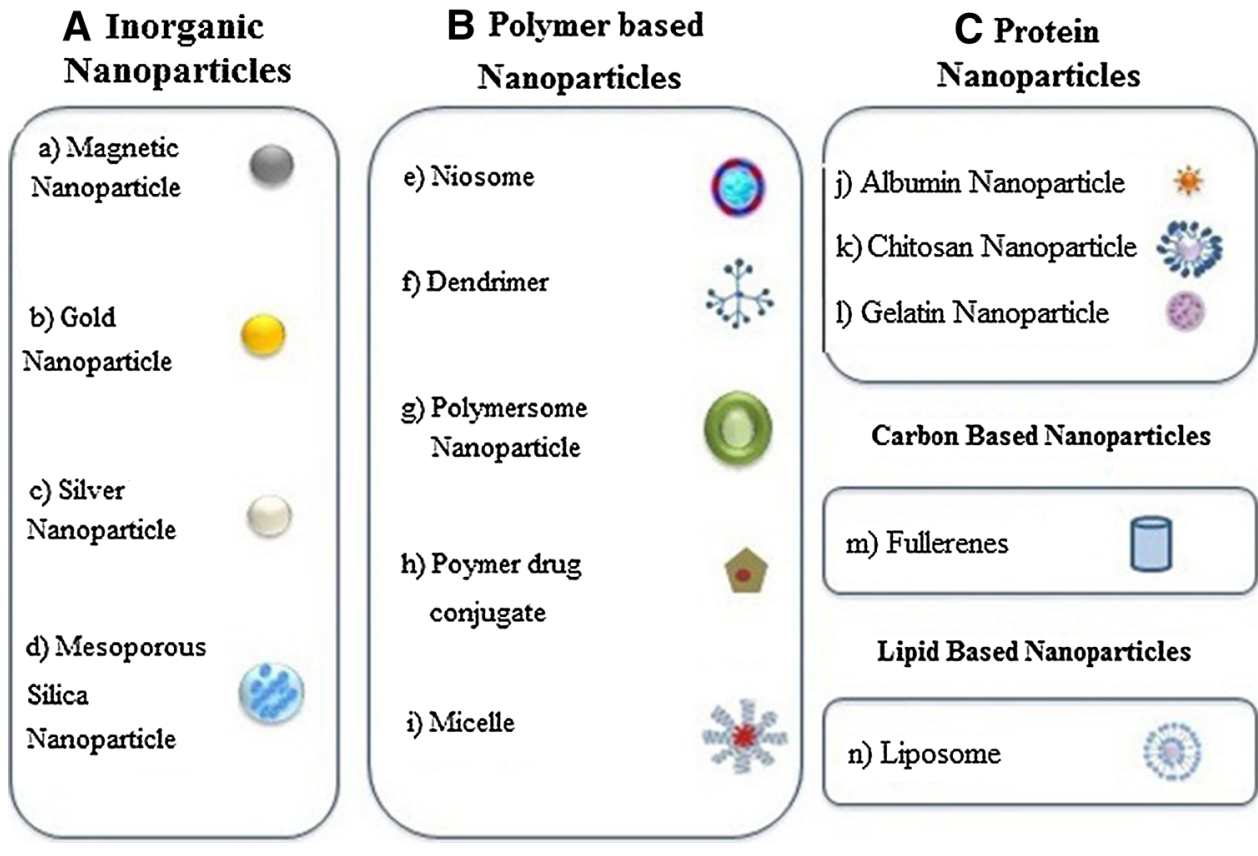


which is followed by chemical functional groups at the exterior terminal surface on radially attached generations $[119,120]$. There is an amplification of molecular size and terminal surface groups with the increase in generations. This allows immense potential for various interactions and hence termed as highly functional. This multivalency enables the creation of various host-guest complexes that offer wide applications. Another application of dendrimers is that they are identical in size to many proteins and biomolecules like insulin, cytochrome $\mathrm{C}$, and hemoglobin. The loading capacity of dendrimers can be customized by the functionalization of different guest molecules onto the surface of dendrimers. Dendrimers have also been found effective against bacterial and viral infection.

\section{Polymeric micelles}

A polymer micelle is a nanoparticle structured by one hydrophilic shell and one hydrophobic core as shown in Fig. 3. It can be divided into two main categories: hydrophobically assembled micelles and polyion-complex micelles. The former ones usually consist of amphiphilic copolymers with a hydrophobic block and hydrophilic block. Balanced between those two blocks in an aqueous medium induces spontaneous formation of nanosized particles [121, 122]. Among the different forms of nanoparticles, in recent years polymeric micelles have gained growing scientific attention. Since their application as nanocarriers in drug delivery system in the 1980s, general studies have shed light on the properties of micelles and introduced them as a promising platform for numerous pharmaceutical applications. They have been evaluated for therapeutic agent (drug, gene, and protein) delivery systems, as well as for diagnostic application. Almost all drug administration routes (parenteral, oral, nasal and ocular) have benefitted from micellar forms of drug in terms of either increased bioavailability or reduction of adverse effects [123].

\section{Lipid-based nanoparticles (liposomes)}

Lipid nanoparticles or liposomes or particularly nanoliposomes which are uni- or multilamellar lipid bilayered vesicles (Fig. 4) act as efficient carriers for small molecules, vaccines, peptides, small and long nucleic acids, and proteins and have led to a rapid advancement in the drug delivery systems. Liposomes were the first nanoparticles applied in medicine since Alec D Bangham and his coworkers described them in 1961 [124]. All nanovectors have a basic structure consisting of a core compartment where the drug is to be delivered, commonly referred to as the "payload" or "cargo", surrounded by a solid matrix or, in the case of liposome, a phospholipid membrane that encapsulates the drug to transport it through the bloodstream. This widens the variety of solubility profiles of drugs that can be delivered and holds hydrophobic drugs in hydrophobic compartments of the nanovector or hydrophilic drugs in hydrophilic compartments. The solid matrix also protects their cargo from plasma enzyme degradation and transports their load across biological membranes and the BBB [125]. Liposomes progress the therapeutic index of new or well-known drugs by enhancing drug absorption, reduction of metabolism, prolonging biological half-life or reduction of toxicity. Properties of the carrier play a vital role in drug distribution rather than physicochemical characteristics of the drug substance only.

There are numerous new methods of liposome preparation based on lipid drug interaction and liposome disposition mechanism including the inhibition of rapid clearance of liposome by controlling particle size, charge and surface hydration [126]. The basic part of liposome is formed by phospholipids, which are amphiphilic molecules
Fig. 3 A diagrammatic explanation of active targeting of tumor with nanoparticles

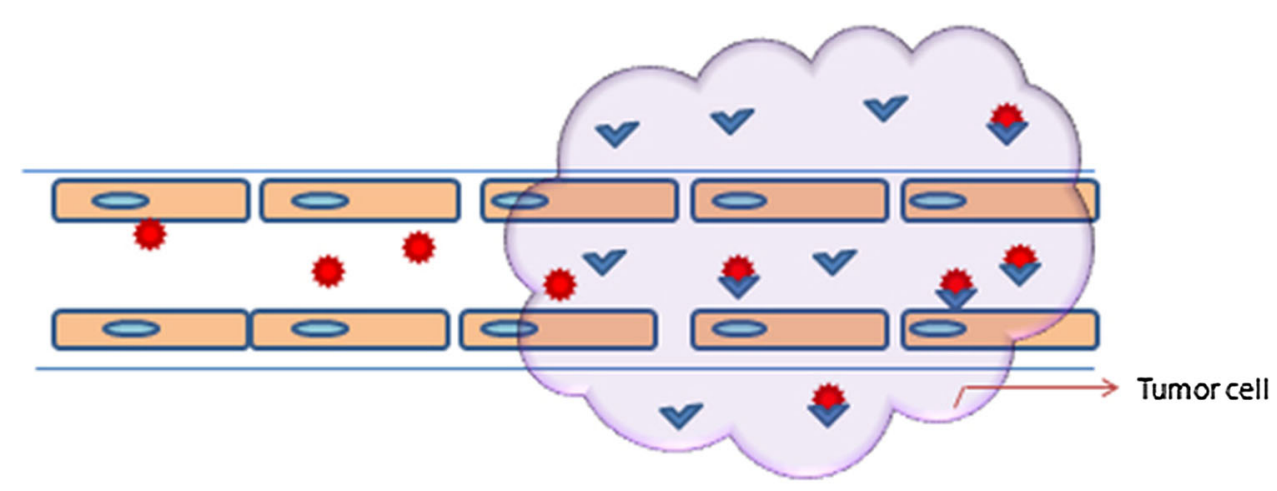

Receptor

Ligand bound

nanoparticle 
Fig. 4 An illustrative representation of passive targeting of tumor with nanoparticles

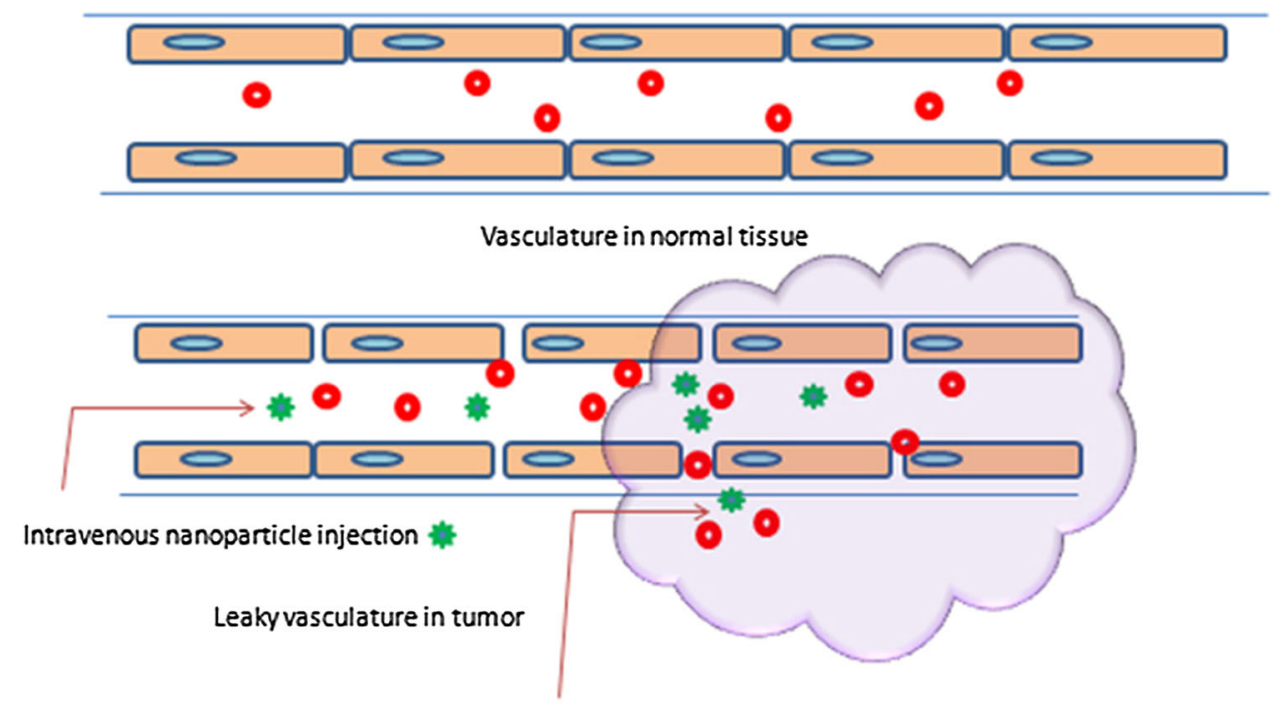

Nanoparticles enters tumor cell passively via EPR effect (having a hydrophilic head and hydrophobic tail). The hydrophilic part is mainly phosphoric acid bound to a water-soluble molecule, whereas the hydrophobic part consists of two fatty acid chains with 10-24 carbon atoms and 0-6 double bonds in each chain. When these phospholipids are dispersed in aqueous medium, they form lamellar sheets by organizing in such a way that the polar head group faces outwards to the aqueous region while the fatty acid groups face each other and finally form spherical/ vesicle-like structures called as liposomes. The polar portion remains in contact with aqueous region along with shielding of the non-polar part.

\section{Hybrid nanoparticles}

In recent days hybrid nanoparticles are developed as nanocarriers that combine advantages from existing systems with well-characterized properties to form lipidpolymer nanoparticles and solid liposomal nanoparticles. The basic parts of hybrid nanoparticles are comprises of at least two different materials, the core and the corona structure. Usually, the core is formed of metallic and polymeric material and is coated with a single or various lipid layers to form a protecting membrane (corona) similar to a liposome or micelle [127]. Combining inorganic or organic nanoparticles to another molecular or macromolecular entity gives rise to new systems exhibiting multiple or synergetic properties. Vaishali Bagalkot and co-workers reported lipid-latex (LiLa) hybrid nanoparticles targeting inflammatory macrophages. The latex core served as a model hydrophobic polymeric template and the lipids provided targeting functionality and colloidal stability. Targeting to inflammatory macrophages was achieved by coating LiLa with phosphatidylserine (PtdSer) and oxidized cholesterol ester derivative cholesterol-9-carboxynonanoate (9-CCN). These lipids, sometimes called "eat-me" signals, are efficiently phagocytosed by macrophages [128]. Proteins of albumin interact with some specific protein receptors in caveolae and caveolae-mediated endothelial transcytosis as well as on tumor cells, are efficiently taken up by these cells and particularly accumulated in tumor cells. Jun Ge et al. have reported protein-polymer hybrid nanoparticles for cancer drug delivery. Self-assembled hybrid protein-polymer nanoparticles from a bovine serum albumin (BSA)-poly (methyl methacrylate) (PMMA) conjugate has also been investigated [129].

\section{Protein nanoparticles}

Natural biomolecules such as proteins are an attractive substitute to synthetic polymers which are commonly used in drug formulations because of their safety. Amphiphilicity of protein makes protein an ideal material for preparation of nanoparticles and helps them to have a better interaction with both the drug and solvent [130]. Protein nanoparticles provide several advantages including biocompatibility, non-antigenic, and biodegradability. These nanoparticles can be prepared under mild conditions by an eco-friendly approach without the use of toxic chemicals or organic solvents. Moreover, protein-based nanoparticles can be customized for surface modifications due to their defined primary structure, and this allows covalent attachment of drugs and targeting ligands. 


\section{Albumin nanoparticles}

Albumin has emerged as a versatile macromolecular protein which acts as a promising carrier for the drug delivery and offers advantage for diagnosis of rheumatoid arthritis, cancer, diabetes, and other infectious diseases [131]. Albumin has several unique features that make it an appropriate vector for targeted drug delivery in oncology. It has been reported to be nontoxic, non-immunogenic, biocompatible, and biodegradable [132]. It is easy to purify and is soluble in water allowing better delivery by injection and thus a perfect candidate for nanoparticles preparation. Therefore, it is a perfect material to fabricate nanoparticles for drug delivery [133-135]. Albumin nanoparticles have high binding capacity with chemicals, proteins/peptides, and oligonucleotides without any severe side effects [136]. It holds bioactive molecules and has shown improved pharmacokinetic properties by providing longer circulation time and more disease-specific accumulation, and they are emerging as a capable theranostic agent [137]. Drugs that are entrapped in albumin nanoparticles can be digested by proteases and drug loading can be quantified. A number of studies have investigated that albumin accumulates in solid tumors making it a potential macromolecular carrier for the site-directed delivery of antitumor drugs [138]. Wellknown market-approved products include fatty acid derivatives of human insulin or the glucagon-like-1 peptide $\left(\right.$ Levemir $^{\circledR}$ and Victoza $^{\circledR}$ ) for treating diabetes, the taxol albumin nanoparticles Abraxane ${ }^{\circledR}$ for treating metastatic breast cancer which is also under clinical investigation in future tumor indications, and Tc-aggregated albumin $\left(\right.$ Nanocoll $^{\circledR}$ and Albures ${ }^{\circledR}$ ) for diagnosing cancer and rheumatoid arthritis as well as for lymphoscintigraphy.

\section{Gelatin nanoparticles}

Gelatin is a versatile natural biopolymer and is considered as GRAS (generally regarded as safe) by US FDA. Its biocompatibility, biodegradability, economical and presence of different target functional groups for binding favor its extensive use in pharmaceutical industries [139]. For the last thirty years, these advantages have led to its application in the synthesis of nanoparticles for drug and gene delivery. Gelatin is a denatured form of animal protein, collagen, which is the major constituent of the corneal stroma, used for ophthalmic applications. It has a basic structure of polyampholyte which consists both anionic and cationic along with hydrophilic group found in an approximate ratio of $1: 1: 1$. It has been recognized that the mechanical properties such as thermal behavior and swelling properties of gelatin nanoparticles depend significantly on the degree of cross-linking between cationic and anionic groups. Gelatin polymer chain is $\sim 12 \%$ negatively charged due to the presence of aspartic acid and glutamic, $\sim 13 \%$ positively charged due to the presence of lysine and arginine, $\sim 11 \%$ of its chain is hydrophobic due to the presence of isoleucine, methionine, leucine, and valine whereas glycine, proline, and hydroxyproline constitute the rest of the polymer chain. Gelatin-based nanoparticles have been fabricated by several researchers using different techniques such as desolation which uses an agent (alcohol or acetone) for dissolving gelatin in aqueous solution. This process dehydrates polymer chain that results in conformational change (stretched to coil). Another simple method for the fabrication of gelatin nanoparticles is reverse phase microemulsion. Here, the aqueous solution of gelatin is further mixed in surfactant-containing solution (SDS or SBES in n-hexane) followed by cross-linking. Gelatin nanoparticles serve as an efficient candidate in delivery and controlled release of the drugs. It has been widely utilized for delivery of both hydrophilic and hydrophobic and anticancer drugs, proteins, vaccine, and genes.

\section{Chitosan nanoparticles}

Chitosan is one of the naturally occurring polymers which bear a chemical formula $\alpha$ (1-4)-2-amino-2-deoxy $\beta$-D-glucan. It is a deacetylated form of chitin which is an abundant polysaccharide present in crustaceans such as shrimps, lobsters, and crabs [140]. It is prepared by removing the shells of these crustaceans and then ground into powder, further processing leads to the production of chitosan. It also occurs naturally in some microorganisms such as fungi and yeast [141]. As it is a cationic polysaccharide and biodegradable polymer, it has been extensively exploited for the synthesis of nanoparticles for restricted delivery of several therapeutic agents. They are inexpensive and simple to manufacture and scale-up and the nanosize enables large surface-to-volume ratio [142]. They are hydrophilic and mucoadhesive in nature which allow them to provide protection to encapsulated drug along with extending its clearance time and stability in the body. Thus, they are relevant to a broad spectrum of drugs, small molecules, proteins and polynucleotides. This mucoadhesive polymer enhances the dissolution rate of these poorly soluble bioactive molecules and also allows their controlled release [143].

\section{Carbon-based nanoparticles}

The potentialities of carbon nanotubes for novel applications in nanomedicine make them very desirable candidate in a series of biomedical applications such as sensors for detecting DNA and protein, diagnostic devices for the discrimination of several proteins from serum samples, and carriers to administer drug molecules, protein or vaccine [144]. Carbon nanotube-mediated drug delivery system has 
raised tremendous importance because of its biocompatibility and supportive substrate. The outer walls of the nanotubes are functionalized by attaching target-specific molecules (e.g., antibodies) and drugs can be delivered to specific targets [145]. Carbon nanotubes are insoluble in all solvents and generate some health issues. The customization of surface with chemical modification renders carbon nanotubes water soluble. They can be functionalized with a vast range of active molecules such as proteins, peptides, nucleic acids, and therapeutic agents. The multiple covalent functionalizations on the sidewall or tips of carbon nanotubes are one of the major advantages for its application in the treatment of cancer therapy. Besides, it is also considered as a promising tool for identifying the expression of indicative biological molecules at early stage of cancer due to its mechanical, electronic, and thermal properties.

Recently, a team of scientists lead by Uday Kishore from Brunel University proved that the interactions between carbon nanotubes and C1q (a starter protein for complement) resulted in anti-inflammatory functions [146]. This theory suggests that either the coating of nanoparticles or healthy tissues with complement proteins is causing to reduce the tissue damage and help in the treatment of inflammatory diseases like Parkinson's, Huntington's, ALS, and Alzhimer's.

\section{Fullerenes}

Fullerenes are a form of carbon nanomaterial which can be functionalized with a wide array of molecules that allows them to gain a prime role on scientific scene. The molecular architecture of fullerenes is arranged in a soccer ball-like structural organization and is crystalline in nature. These are also known as buckyballs, discovered in 1985 among the detritus of laservaporized graphite [147]. Given their unique structure and properties, these carbon-based compounds have proved to become one of the most promising nanomaterials capable to diagnose, monitor and treat certain medical conditions. Unlike other molecules that have applications as cancer drug delivery vectors, fullerenes are not fragmented in the body and are egested intact. This feature can be important for some cancer treatment compounds that have toxic effect to healthy cells [148]. Fullerene is competent to fit inside the hydrophobic cavity of HIV proteases. This inhibits the availability of substrates to the catalytic site of enzyme. In addition, it can be used as radical scavenger and antioxidant [149].

\section{Potential targeted drug delivery of nanoparticles}

During the last three decades, therapeutic nanoparticles have advanced significantly and revolutionized the scenario of pharmaceutical industry leading to the development of various clinical formulations. This has eventually improved patient compliance and convenience. By virtue of nanosize and other improved physicochemical properties, nanodrugs and carriers are capable to deliver drug at the targeted site of disease. Targeted drug delivery means specific interaction between drug and its receptor at the molecular level. The basic requirement of targeted drug delivery system is design of nanocarriers to carry the drug from the site of administration to the target site and release the therapeutic payload with minimum loss of their volume and activity in blood circulation. Second, drugs should only kill the specific diseased cells without adverse toxic effects to healthy tissue. Third, the vector should protect the drug from enzymatic reactions and other environmental factors. Drugs can be conjugated with aptamers, proteins, peptides, antibodies to reach their intended target. The prime advantage of this drug delivery system is that the pharmacokinetic behavior of the nanocarrier does not depend on the whole nanosystem rather than on the drug itself. The targeting is facilitated using two modes: passive and active targeting of drugs.

\section{Passive and active targeting of nanoparticles}

Passive targeting occurs in our body naturally. As the hormones and growth factors have natural tendency to reach the target receptor, in the similar way the drugs are also carried to the site of action by physiological circumstances. Passive targeting involves the formulation of a drug carrier complex in such a way that it cannot be excreted from the body by defense mechanisms like excretion, opsonisation, and metabolism followed by phagocytosis. The drug complex system keeps circulating in the blood stream and allows itself to be taken to the target site by physiological factors such as warmth, $\mathrm{pH}$ or morphology of the drug [150]. Several factors have to be taken into concern for the transformation of a drug molecule into a device that can circulate in blood and bind only to the intended target receptor. Such factors are its surface charge, molecular weight, hydrophobic or hydrophilic nature of its surface and morphology. Passive targeting is achieved by incorporation of therapeutic agent into drugs or macromolecules, or coupled to macromolecule or nanoparticles that can passively reach the target site. Though passive targeting has been observed to achieve significant output, the pursuit of better control over drug modulation has led to further research on active targeting. The therapeutic agent in active targeting is acquired by conjugating the therapeutic agent or carrier system to a cell-specific ligand or tissue. It involves attachment of the medicative agent to carrier protein, or antibody, or a ligand which can allow it to go and meet and complex with receptor-bound cell. Nanocarrier complex can recognize 
and bind to the target cells through ligand-receptor interactions by the expression of epitopes or receptors on the cell surface. Those receptors are highly expressed on tumor cells but not on normal cells for achieving high specificity.

\section{Nanoparticle targeting in cancer cells}

In recent years, cancer is one of the leading causes of mortality rate. One of the major causes behind rise in death rate is inability to deliver the drug to target-specific site without inducing any toxicity to the normal tissue. Current treatment regimes of cancer mainly rely on chemotherapy. So there is an essential need to develop a drug delivery system which could overcome biological barriers and selectively target the cancerous cell. The development of targeted nanotechnology-based drug delivery improves the drug/gene delivery and overcome multiple physiological, physical and biological barriers which are associated with conventional chemotherapy. For instance, nanoparticles via either passive or active targeting enhance the intercellular concentration of drugs/genes in cancer cells while avoiding adverse effect to normal cells. In addition, targeted nanoparticles can be fabricated as either $\mathrm{pH}$-sensitive or temperature-sensitive carriers. The $\mathrm{pH}$-sensitive drug delivery systems are controlled to deliver and release drugs within the more acidic environment of the cancer cells or within cancer cells [151]. The temperature-sensitive system are designed to release drugs with changes in temperature locally in the tumor region provided by sources such as ultrasound waves, magnetic fields and so on. Therefore, a hybrid approach of mutual therapy such as chemotherapy and hyperthermia has come up with promising outcomes [152].

Most nanoparticles usually gather in tumors due to the enhanced permeation and retention (EPR) effect. Blood vessels of the tumor are permeable due to defected angiogenesis. As a result, the nanoparticles accumulation occurs in tumor. Again, lymphatic drainage of tumors is dysfunctional that it helps the nanoparticles to remain in tumor for longer period of time and allow localized nanoparticle disintegration and release of the drug in the vicinity of tumor cells. The active targeting mechanism is characterized by increased cellular uptake and increased tumor retention due to high specific connections between the targeting ligand and certain tissues or cell surface antigens. These ligands can recognize and bind to receptors, or complementary molecules, found on the surface of tumor cells. When such targeting molecules are conjugated to drug delivery nanoparticle, more of the anticancer drug intends to enter the tumor cell, and increase the efficacy of treatment along with reduction of toxic effects on surrounding healthy tissue. In Table 2 are shown various nanocarriers evaluated to deliver therapeutic agents to defected cells.

\section{Nanoparticle targeting in Alzheimer's disease}

Alzheimer's disease (AD) is one of the most widely recognized neurodegenerative disorders of the elderly with almost incurable and limited treatment solutions with nonspecific suspected causes. Increasing research investigation indicates that the overproduction of amyloid beta $(A \beta)$ and incapacity of $A \beta$ peptides to be cleared from the brain is one of the causes of degeneration of nervous system. The cumulative deposition of this protein results in self-aggregation to form toxic oligomers, neurofibrillary plaques, and tangles [161]. The plagues are made by single molecules of $\mathrm{A} \beta$ cluster. These disrupt cell-to-cell signaling at synapses and stimulate the immune system. On the other hand, neurofibrillary tangles are formed when tau protein required for the maintenance of transport networks of cell breaks down. Consequently, this causes death of brain cells due to the scarcity of essential protein and nutrients. Currently leading working theory explains plaques and tangles described above as the major cause of cell death and tissue loss found in an $\mathrm{AD}$ brain, though the theory is yet to be indisputably confirmed. As a result, brain cells slowly disintegrate progressively invading different parts of the

Table 2 Examples of nanocarrier system to deliver therapeutic agents into targeted cells

\begin{tabular}{lllll}
\hline Ligand/Target & Nanosystems & Drug & Cell/tumor model & References \\
\hline AS1411 DNA apt & Nucleation/Liposomes; PEG-PLGA NPs & Cisplatin; PTX & MCF-7; C6 glioma cells & [153] \\
CD44 & PLGA-nanoparticles & Salinomycin and Paclitaxel & MCF-7; MDA-MB-231 & [154] \\
PSMA & Superparamagnetic iron oxide nanoparticles & Docetaxel & PC Cell lines & [155] \\
CXCR4 & Nanoparticles; PLGA/TPGS & Epirubicin & HepG2 & SW620 \\
EpCAM & Aptamer-mesoporous silica nanoparticles & Doxorubicin & Panc-1 & [156] \\
EGFR & Gelatin nanoparticles-PEG & Gemcitabine & Molt-4 \\
T cell & Gold nanoparticles-sgc8c aptamer & Daunorubicin & A549 \\
CXCR4 & PLGA-nanoparticles & Doxorubicin & & [158] \\
\hline
\end{tabular}


brain. This creates some characteristic changes that significantly distinguish the different stages of Alzheimer's. A $\beta$-induced mitochondrial dysfunction due to abnormal production of ROS has also been known to be a probable cause of $\mathrm{AD}$.

The amelioration of Alzheimer's with AD drugs is a very promising area of research and several bioactive molecules reach the clinical trials but they ran out of success. One of the major reasons of this failure is restricted access of drug through BBB. The BBB is anatomically defined as the cerebral microvascular endothelium, which bears different structural organization of vascular beds. It has few alternate transport pathways and tight cell-cell junctions. This ceases the pinocytotic activity and significantly decreased number of intracellular fenestrae. The brain endothelial cells express high levels of active influx/efflux membrane transport proteins including P-glycoprotein, Multidrug Resistance Protein-I, Breast cancer Resistance Protein and have additional degrading enzymes which enable selective permeability of molecules [162]. Nanoparticles have been explored for controlled and target-specific delivery of the drug that can transport across the BBB and increase the uptake of suitable drugs in the brain. Biodistribution of NP drug can be restricted from phagocytic cell populations because the smaller particles can be captured by Küpffer cells. Nanoparticles retain the drugs in blood circulation for a longer time, which facilitates drug ability to interact with specific molecules expressed on the luminal side of BBB endothelial cells. A concentration gradient formed by increased retention of NPs in the brain-blood capillaries coupled with adsorption to capillary walls increases the transport of NPs across the endothelial cell layer for drug delivery. It also solubilizes lipids of endothelial cell membrane resulting in membrane fluidization which in turn opens the tight junction between endothelial cells. This leads to enhanced permeation of the drug through nanoparticulate-mediated endocytosis by endothelial cells followed by drug release within these cells of the brain transcytosis of the NPs with bound drug across the endothelial cell layer, phagocytosis and carrier-mediated transport or by absorptive transcytosis. In this regard, the proper surface functionalization of NPs' surface (coating or conjugation) is crucial matter of concern. A few examples of surface-customized NPs for this purpose contain thiamine-coated NPs, transferrin-coated nanoparticles, PEGcoated NPs, etc. NPs can be prepared as polymer (chitosan, heparin albumin, etc.) conjugate or could be a fullerenolcytotoxic conjugate. The additional methods like LipoBridge technology (facilitates temporary opening of tight junctions of BBB) liposomal technology, angiopep (nineteen amino acids containing peptide vector) system shall be included [163]. Recently a group of researchers investigated therapeutic ceria $\left(\mathrm{CeO}_{2}\right)$ nanoparticles can remove ROS in mice genetically modified to show the symptoms of $\mathrm{AD}$, strong and recyclable ROS scavengers that shuttle between $\mathrm{Ce}^{3+}$ and $\mathrm{Ce}^{4+}$ oxidation states [164]. The nanoparticles were targeted to the mitochondria using a compound called triphenylphosphonium.

\section{Nanoparticle targeting in diabetes mellitus}

Diabetes mellitus is a group of metabolic disorder distinguished by high blood glucose level in blood due to lower binding efficiency of insulin on their cell surface receptors or due to lower insulin secretion by the cells. Type 1 diabetes results from the inadequacy of $\beta$ cells that are destroyed by the immune system. Type 2 diabetes is mainly involved with $\beta$ cell dysfunction and insulin resistance. Both type 1 and 2 diabetes share a common characteristics, i.e., $\beta$ cell mass reduction [165]. The major obstacles associated with diabetes management are selfmonitoring of insulin injections and blood glucose levels. The development of nanomedicine has provided a new facet for the treatment of diabetes with glucose nanosensors, carbon nanotubes, quantum dots, nanopumps, oral insulins, microspheres and artificial pancreas.

The monitoring and real-time tracking of blood glucose levels can provide more accuracy since glucose nanosensors are employed in implantable devices. This can also provide the basis for glucose-responsive nanoparticles that can mimic the body's physiological needs for insulin in a better way. The layer-by-layer method is associated with the principle of electrostatic charge of sporadic layers of positively and negatively charged polymers. The polymers are fabricated as miniaturized films with customizable, biocompatible and flexible pores. These bilayers might be permanently fixed in subcutaneous tissue as a "smart tattoo". Other than that, semi permeable capsules allow the glucose to pass from interstitial fluid while protecting the sensor. Insulin is transported via biodegradable polymeric carriers which have a matrix surrounded by nanoporous membrane containing grafted glucose oxidase. As blood glucose level rises, it triggers a change in the surrounding nanoporous membrane and results in biodegradation and consequently insulin delivery. The glucose/glucose oxidase reaction decreases the $\mathrm{pH}$ level in the delivery system's microenvironment. This in turn increases the swelling of polymer system, and enhances release of insulin.

\section{Nanoparticle targeting in Parkinson's disease}

Parkinson's disease (PD) is a neurodegenerative disease, which is caused by the loss of dopaminergic neurons of substantia nigra. This decrease in dopamine is correlated 
with the motor impairment and eventually leads to tremor, bradykinesia and rigidity [163]. Patients are generally treated with dopamine agonist drugs such as amantadine, budipine, levodopa but these drugs do have unfavorable benefit/side effect profile ratio as well. Levodopa remains as the gold standard of treatment. The efficacy of these drugs fades away after some years as the side effect restricts its long-term use. Moreover, the bioavailability of orally administered drugs depends on several factors when it reaches the dopamine receptor such as gastric $\mathrm{pH}$ and dietary protein. Another administration routes such as intranasal, rectal, sublingual or pulmonary did not show any satisfactory result because none could maintain a continuous stimulation of dompaminergic drug. Miniaturization of a device to nanoscale and incorporation of drug for its continuous release have become major challenges. Researchers are carrying out several approaches with dopamine infused in liposome and are testing its efficacy on rat model of PD. Dopamine conjugated on the surface of chitosan nanoparticle administered in rat model has resulted in less cytotoxic long-term release than dopamine alone. Other than that polyethylene imine-grafted chitosan nanocarriers for gene delivery approach have also been studied [166, 167]. Recently theranostic device such as LDOPA coated with manganese oxide which can act as a dual MRI contrast agent as well as drug delivery agent is a promising area of interest for PD treatment [168]. Polymers such as polyvinyl pyrrolidone-poly (acrylic acid) (PVP/ PAA) and PLGA have been used for the synthesis of NP and investigation carried out on animal model has shown better results in comparison to free dopamine alone. Administration of biodegradable nanodrug to the targeting molecules of PD which include neurotrophic factors such as GDNF-MPs in rat model and monkey model has shown better structural and functional recovery of Parkinsonian monkey.

\section{Nanoparticle targeting in angiogenesis}

Angiogenesis involves the maturation and growth of new blood vessels for the delivery of oxygen and nutrients throughout the body. Thus, angiogenesis plays a substantial role in growth and development as well as in pathological diseases such as rheumatoid arthritis, exudative age-related macular degeneration, eye diseases, ischemic heart disease, metastasis and tumorigenesis. It ensures the normal embryonic vascular development of all vertebrates, as well as regulates physiological processes such as wound healing and menstruation in adults. The whole process of angiogenesis is controlled and triggered by chemical signals in the body. These chemical signals function for repairing both injured blood vessels and the re-growth of new blood vessels. Formation of the blood vessels is obstructed by chemical signals called angiogenesis inhibitors. This stimulatory and inhibitory action of these chemicals signals prevails so that blood vessels form only and when they are needed. Angiogenesis is the result of chemical signal imbalance. Angiogenesis is initiated when endothelial cells are stimulated by specific molecules that bind to angiogenic receptors and promote a signaling cascade. This activation in turn leads to proliferation of the endothelial cells, which then gather to form the new vascular structures. The whole process is regulated by signaling cascade pathway with the involvement of pro-angiogenic factor and antiangiogenic factor. Among the several growth factors involved in angiogenesis including platelet-derived growth factor (PDGF), vascular endothelial growth factor (VEGF), fibroblast growth factors (FGF), epidermal growth factor (EGF), VEGF has been identified as the imperative one.

In recent years, many researches have been used for targeting angiogenesis. Furthermore, nanovehicles have been employed as theranostic agents for anti-angiogenesis therapy and imaging of the tumor vasculature. Both synthetic and natural nanoparticles such as polymeric conjugates, liposomes, micelles, and polymeric nanoparticles, and synthetic organic nanoparticles such as carbon nanotubes, polyhydroxylated fullerenes, dendrimers, inorganic nanoparticles of gold, silver and iron oxide, quantum dots, viral capsids and ferritin have been utilized for targeting angiogenesis.

\section{Nanoparticle targeting with macrophages to control inflammation}

Macrophages participate and regulate inflammatory process through the autoregulatory loop. In diseases such as atherosclerosis, obesity myocardial infarction, and cancer macrophage plays a major role [169, 170]. It has been studied that the inflammatory macrophages in atherosclerotic plaque is closely associated with plaque rupture. In case of obesity, macrophages penetrate the adipose tissue during weight gain and give rise to local and systemic inflammation consequently causing type 2 diabetes mellitus and insulin resistance (IR) [171]. Moreover, many of the pathogens acquire resistance and manage to reside within the macrophage itself by avoiding phagocytosis and lysosomal tolerance. Therefore, to circumvent the diseaseassociated side effect and low solubility of drugs, targeted delivery of nanodrug holds promise for the cure of disease. Still the targeted delivery of drug molecules specifically to macrophages to combat diseases remains a challenge. Fabrication of new carrier systems with intensive studies have been performed vigorously [172-174]. These 
Fig. 5 A diagrammatic representation of targeting macrophage with nanoparticles

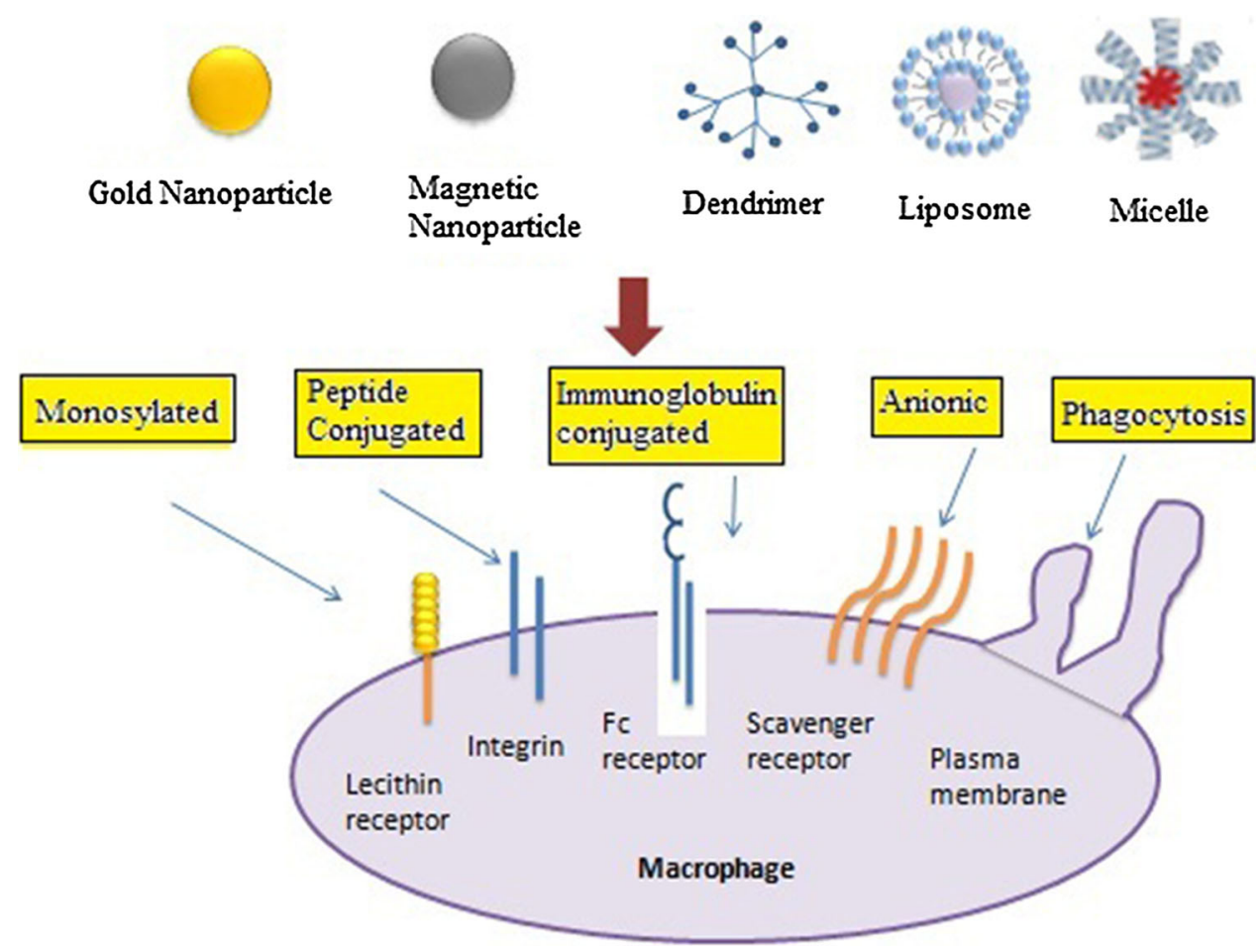

treatment regimes are primarily based on the patternrecognition receptors which are present on the cell surface of macrophages such as the family of scavenger receptors, examples include Fc and complement receptors, mannose receptor Fig. 5 [175-180], which are used by macrophages for phagocytosis [181-184]. In many cases, receptors for amyloid- $\beta$ peptide [185], high-density lipoprotein [186] and hyaluronic acid [187] were utilized as a candidate for targeted delivery of drug. Development of target-specific delivery systems may reduce the amount of therapeutic agent needed to obtain a clinical effect, which may effectively cease drug-induced toxicity and other side effects. The incorporation or encapsulation of drug molecules into carrier systems may also protect against drug degradation or inactivation en route to the macrophages. Polyalkylcyanoacrylate (PACA) nanoparticles have been used as a vector to deliver antileishmanial drugs into macrophages. This nano-based carrier system did not induce any interleukin-1 release by macrophages [188]. Research teams are carrying on their study targeting macrophage infections in chronic diseases with genes also. Recently, Aouadi et al. has investigated the efficacy of $\beta$-glucan-encapsulated siRNA particles as oral delivery vehicles for targeting macrophages. These particles can silence the Map $4 k 4$ gene and protect animals from lipopolysaccharide-induced lethality [189]. Still, this approach involved a complex formulation process and multiple components and utilized cytotoxic polyethyleneimine (PEI), which may cause adverse effects and decrease their reproducibility. On the other hand, Huang et al. simplified the encapsulation process by direct absorption of protein antigen inside the glucans. These particles incited strong immunologic responses in vivo as an immune adjuvant [190]. However, the direct absorption process may release the absorbed protein from the carrier too quickly. Therefore, new strategies for protein encapsulation inside glucan particles are necessary for the efficient use of $\beta$-glucan particles as targeted protein-delivery systems for macrophages.

\section{Nanoparticle targeting with inflammatory molecules}

For a few decades, the suppression of inflammation and inflammatory responses has been a prime area of focus because nonresolving inflammation is not only a response cause of many degenerative diseases, including obesity, atherosclerosis, asthma, chronic, obstructive pulmonary disease, rheumatoid arthritis, inflammatory bowel disease, cardiovascular neurodegenerative diseases, cancer, and sepsis, but it also plays a significant role in their pathogenesis [191]. A precise and sensitive exposure of the site of inflammation may contribute producing a wide-ranging impact on the pro diagnosis and treatment of the disease. When inflammation is induced, a number of adhesion molecules are upregulated in endothelium. These molecules are interacted by immune cells using counter adhesion molecules such as integrins, which adhere to 
endothelium and initiate diapedesis [192]. Researchers are seeking to exploit nanoparticles for the detection and treatment of inflammation involving antibodies or peptides specific to adhesion molecules such as vascular cell adhesion molecules (VCAM) [193-195], intracellular adhesion molecules (ICAM) [196-198], collagen [199], selectins [200], and the immunoglobulin super family. Among these molecules, ICAM has gained a particular interest because of its localized expression upon inflammatory signals, which can serve as a marker for inflammation despite its constitutive low level expression [201, 202]. The ICAM family consists of five members, designated ICAM-1 to ICAM-5, which binds to the leucocyte integrins CD11/ CD18 during inflammation and in immune responses. Again, ICAMs may also exist in soluble forms in human plasma, due to proteolysis and activation mechanisms at cell surfaces. VCAM on the other hand, can bind to leucocyte integrin VL-4 (very late antigen-4) to recruit leucocytes to sites of inflammation. A team of scientists from Harvard Medical School in Boston has developed a novel strategy with liposome-based targetable nanoparticle loaded with cell cycle regulatory molecule cyclin D1 siRNA. Si RNA has also been conjugated with protamine, a positively charged protein for the better delivery of nucleic acid. Monoclonal antibodies such as F1B504 have been reported to bind integrin when attached to hyaluronan liposome nanoparticles.

\section{Mechanisms of drug resistance}

\section{Multiple drug resistance (MDR)}

The development of multiple drug resistance in cancer treatment to chemotherapeutic agents refers to the capacity of cancer cells to survive or become resistant from therapy involving a wide variety of drugs. It is a state of resilience development against functionally or structurally related drug. The progressive development of MDR in cancer is one of the prime factors responsible for incurability of disease. Demand for urgency to develop a clinical procedure for overcoming the multidrug resistance in cancer arises. The resistance of tumors not only progresses against a single cytotoxic drug, but also occurs as a result of crossresistance in a whole range of drugs with different structural and cellular targets. Once MDR appears, application of high doses of drugs to overcome resistance becomes ineffective, toxic effects appear and resistance is further generated [203-205]. It affects a major group of cancers patients including ovarian, breast, lung, and lower gastrointestinal cancers and resistance exists against every effective anticancer drug. Resistance involves numerous mechanisms including decreased drug uptake, increased drug efflux along with reduction of intracellular drug concentration, activation of detoxifying systems, activation of altered DNA repair mechanisms, evasion of drug-induced apoptosis, induced emergency response genes to impair apoptotic pathway, etc. These mechanisms can be characterized as non-cellular or cellular based on the factors contributing to MDR development [206].

\section{Non-cellular MDR}

These mechanisms generally presume for certain types of cancers which show natural or inherent resistance to chemotherapeutic drug at the initial exposure. MDR may occur as a consequence of non-cellular mechanisms arising from solid tumor growth in vivo. The solid tumors suffer from poor vascularisation, hypoxia and accessibility to the drug. It limits the drug-induced cytotoxicity and also reduces the friction of vulnerable cells due to presence of non-cycling tumor cells resistant to proliferation-dependent drugs [207]. Such growth environment favors the proliferation of cancer cells to proliferate which is markedly different from that of the normal cells. Inadequate nutrition and hypoxia due to poor vasculature resulting in lactic acid accumulation helps to generate resistance to cancer cells against drugs as the cellular uptake of drug needs $\mathrm{pH}$ gradient [208].

\section{Cellular MDR}

Cellular MDR mechanisms may be broadly classified as non-transport-based or transport-based mechanisms. Nontransport-based cellular MDR mechanisms deal with the involvement of enzyme systems that modulate the desired activity of the drug without altering its effective concentration inside the cell [209]. This can be observed in the treatment of acute myelogenous leukemia with cytarabine (AraC), a nucleoside drug. This drug is activated after a series of multiple phosphorylation events when it is converted to AraC-triphosphate [210, 211]. Mutation in this metabolic pathway or down-regulation can hinder the activation of $\mathrm{AraC}$, which ultimately leads to $\mathrm{AraC}$ drug resistance. Another important example for drug activation and inactivation includes glutathione- $S$-transferase (GST) super family, the cytochrome P450 (CYP) system, and uridine diphospho-glucuronosyltransferase (UGT) super family [212].

The transport-based cellular mechanism of MDR mainly involves the efflux of drug from the cell by various energydependent membrane transport proteins, which do not allow the drug to reach therapeutic concentrations inside the cell [213]. ATP-binding cassette (ABC) transporters are a family of proteins that stimulate MDR via ATP-dependent drug efflux pumps [214]. Overexpression of ATP- 
binding cassette $(\mathrm{ABC})$ transporters has been shown to be responsible for MDR [215].

At least only three human ABC transporters among 48 have been studied broadly and have been found that MDR is closely associated with anti-inflammatory, anticancer, and anti-viral drugs [216]. The multidrug resistance has also been linked to the breast cancer resistance protein (BCRP or ABCG2), protein 1 (MRP1 or ABCC1) and P-gp (P-glycoprotein), also called transmembrane small molecule pump (ABCB1). Transport proteins of the ABC superfamily such as P-glycoprotein, multidrug resistanceassociated protein-1 (MRP1) its homologs MRP2-6 and the breast cancer resistance protein (BCRP) [217-219] have been characterized. These proteins are overexpressed in malignant cells and pump the anticancer drugs out of the cell which decreases the intracellular levels of the drug necessary for effective therapy.

\section{P-Glycoprotein (P-GP)}

P-Glycoprotein (P-gp) has been studied as a poor prognostic factor rather than a specific determinate for the resistance against chemotherapeutic drugs and it was described by Juliano and Ling first in 1976 in Chinese hamster ovary cells selected in a culture for colchicines resistance, a phenotype of cancer [220]. P-gp is one of the most widely studied MDR family members not only for its function in extruding various cytotoxic compounds out of the cells but also for modulation of chemokines, cytokines, and other small peptide secretion [221]. The multidrugresistant tumor cells are known to express glycoprotein unique to the cell surface. The protein posses ATPase activity and can inhibit drug efflux along with reverse resistance to an astonishing array of anticancer drugs. It is overexpressed in tumor cells which protects the cell by pumping out anticancer drugs, and gives rise to multidrug resistance. The efflux pump P-gp functions for transporting various xenobiotics out of cells using ATP. The g-protein encoded by the MDR1 gene is also called the ATP-binding cassette (ABC) protein. MDR1 has a characteristic promiscuous substrate specificity, which allows its ability to transport structurally diverse compounds across the cell membrane, including hydrophobic compounds. However, studies state it could be correlated with some drugs as P-gp substrates such as chemotherapeutic agents, $\mathrm{Ca}++$ channel blockers, $\beta$ Adrenoreceptor antagonists, steroids, cardiac drugs, immunosuppressant, opioids, lipidlowering agents, Human Immuno Deficiency Virus (HIV) antiemetic, antibiotics, protease inhibitors and histamine $\mathrm{H} 1$ receptor antagonists. $\mathrm{P}-\mathrm{gp}$ is also expressed in many normal tissues, such as kidney, liver, intestine, placenta, and BBB. In the brain, P-gp acts as a neuroprotective agent and prevents harmful compounds from reaching the brain.

\section{Cancer stem cells and drug resistance}

Cancer stem cells are cells with a small fraction of tumor cell bearing the characteristics of stem cell. They are capable of self-renewal and regenerate into a tumor. Cancer stem cells are naturally acquiring resistance to chemotherapy by a range of mechanisms through their specific morphology, their ability for DNA repair, their quiescence, overexpression of anti-apoptotic proteins, and ABC-transporter expression [222, 223]. Consequently, some tumor cells are killed and some survives for the regeneration of another tumor. In case of acquired resistance, tumor stem cell with drug-resistant variants gives rise to close descendants and produces a population of multidrug-resistant tumor. This can commonly occur with patients having history of recurrence of cancer following chemotherapy. This mechanism is also applicable for cancer stem cells where mutations accumulate over time, produces the long-term effect of exposure to carcinogenic agents and grants drug resistance to their abnormally developing offspring. Thus, the intratumoral heterogeneity of cancer cells presents a major challenge to the development of effective cancer therapies [224-227]. In 1997, Bonnet and Dick identified a single cell isolating from a bulk cancer cell population. This single cell initiated cancers in an immune-compromised animal model that replicated the cellular heterogeneity of the parent pathology. Extensive research demonstrated that acute myelogenous leukemias (AML) could be initiated in NOD/SCID mice by transplanting a rare $(<0.2 \%$ of whole cell population) CD34 ${ }^{+} / \mathrm{CD} 38^{-}$cell from human donors. Only cells which display these unique markers were capable of engrafting and generating the cellular diversity evident in human AML [228].

Chemo- or radiotherapy was also used to explain the recurrence of increasingly invasive and malignant tumors in context of survival and accumulation of cancer stem cell drug resistance. Detection of cancer stem cell in a small subpopulation of cancer cells can be characterized by specific phenotypes, for example, CD $44^{+} / \mathrm{CD} 24^{-}$in solid tumors, or $\mathrm{CD} 133^{+}$in other tumors, and $\mathrm{CD} 34^{+} / \mathrm{CD} 38^{-}$in leukemia cells.

\section{Insulin resistance}

Insulin resistance plays a major role in the pathogenesis of type 2 diabetes and the metabolic syndrome. Insulin resistance is defined as a state in which the body loses its ability to respond to insulin which is a key factor in the development of diabetes mellitus. Research has stated that the development of insulin resistance is closely associated with obesity, cardiovascular disease type 2 diabetes mellitus along with immune cell infiltration and inflammation 
[229]. In this condition the muscle, fat, and liver cells are likely to have a decreased responsiveness and cannot absorb glucose easily from the bloodstream and do not respond properly to insulin. As a result, the body demands higher levels of insulin to provide glucose entry into cells. To balance this demand, the beta cells in the pancreas also produce more insulin. As long as the beta cells are able to produce enough insulin to overcome the insulin resistance, blood glucose levels stay in the healthy range. Beta cells have two insulin receptors. If one of them gets insensitive to insulin, the other insulin receptor is activated by a different pathway, which leads to beta cell proliferation.

Studies have led to explore that a wide range serious illness, including obesity, dyslipidemia, glucose intolerance and even cancer are correlated with insulin resistance [230]. Insulin-mediated action involves a series of cascade of the signaling pathways. Initially, insulin binds to its receptor and elicits the receptor autophosphorylation. This in turn activates the receptor tyrosine kinase and results in tyrosine phosphorylation of insulin receptor substrates. The phosphorylation of insulin receptor substrates further promotes the activation of phosphotidylinositol 3-kinase and, subsequently stimulation of Akt and its downstream mediator AS160. All of the above biomolecules are required for the stimulation of glucose transport by insulin induction.

\section{Brain disease drug resistance}

Resistance to drug treatment is considered as a prime obstacle in the therapy of many brain disorders, such as epilepsy, brain tumor, schizophrenia, Parkinson's, Alzheimer's, and depression. Degenerative brain diseases affect by causing memory and cognitive dysfunction and behavioral problems. Drugs developed so far for treating Alzheimer's disease temporarily help in restoration of memory but they do not cure the underlying cause of the disease. Thus, there is a need for the fundamental treatment or suppression of disease progression. Current treatment regimes are inadequate to affect disease to ameliorate the brain degeneration as the therapeutic agents cannot reach the BBB in the nervous system and retain the ability to achieve targeted delivery to appropriate brain or spinal cord subregions.

The BBB controls the cerebral homeostasis and protects the central nervous system (CNS) against the toxicity of many xenobiotics and pathogens. BBB selectively screens the biochemical structural, physicochemical characteristics of the solutes at its periphery and thus affords barrier selectivity in the passage of desired molecules into the brain parenchyma. Efflux transporters are expressed in all cells of the BBB that helps to protect them from exogenous or endogenous toxic substances. Most of these transporters belong to the superfamily of ATP-binding cassette (ABC) proteins, which drive the cellular extrusion of many therapeutic drugs with different structures and clinical uses. To enter the brain parenchyma and cross the BBB the substrates in the systemic circulation, molecules must either diffuse passively or transport actively across this barrier. Drug resistance in brain diseases is contributed by multidrug transporters in two ways. First, they restrict access of many drugs and enhance drug extrusion from the brain through their expression in the BBB and blood-CSF barrier. So the drugs cannot reach the brain with sufficient concentration. Second, in some brain diseases, intrinsic or acquired overexpression of multidrug transporter in the $\mathrm{BBB}$ are overexpressed and this limits drug to penetrate into the target tissue [231]. Mahar Doan et al. analyzed 18 different drugs with physiochemical properties used to treat CNS and non-CNS disease for identification of characteristics that correlated with efficacy in the treatment of brain diseases. Research revealed that CNS drugs were found to have fewer positive charges, fewer hydrogen bond donors, lower polar surfaces, greater lipophilicity with reduced flexibility-properties that indicate enhanced membrane permeability [232].

\section{Rheumatoid arthritis drug resistance}

The pathophysiology in rheumatoid arthritis involves cells of immune system ( $\mathrm{T}$ lymphocytes and macrophages) as the causes of resistance [233]. The possible underlying mechanisms of drug resistance may include defective cellular uptake, increased drug extrusion, alterations in intracellular drug activation, target inhibition, processes, downstream of target inhibition, impaired drug delivery to target cells or an amalgamation of these features. The mechanisms of resistance to DMARDs (disease-modifying anti-rheumatic drugs) might be similar to those of resistance to anticancer and antimicrobial drugs and may be associated with the alteration in and intracellular metabolism and drug efflux transporters as well as genetic predisposition. Most of the patients discontinue conventional DMARDs courses within two years either due to toxicity or due to the lack of efficacy. It has been reported that $24 \%$ of patients suffer from lack of responsiveness to certain DMARDs and a further 25\% may stop responding after an initial period of response. Recently, a study by Morgan on a small population of rheumatoid arthritis patients has demonstrated that the primary unresponsive to DMARDs may be, possibly due to MDR related mechanism [234]. Still the role of $\mathrm{ABC}$ transporters for determining the efficacy of DMARDs in patients with rheumatoid arthritis is unclear. Among the MDR family members P-gp is one of the most widely studied for its function in modulating inflammation tuning the secretion of chemokines, 
cytokines, and other small peptides and evicting various cytotoxic compounds out of the cells.

\section{Drug resistance overcome by nanotechnology and nanocarrier-based drug delivery}

MDR offers major obstruction towards the successful treatment of non-infective degenerative diseases. Numerous strategies are on for last few decades to overcome the drug resistance which includes inactivation of MDR-related mRNA in targeted approach, monoclonal antibody usage against extracellular epitope of MDR efflux transporter, development and modification of new chemotherapeutic drugs that are not recognized by MDR efflux transporter and hybrid approaches as well. Still there is a pressing need for combating the drug resistance in degenerative diseases. An innovative approach for overcoming MDR is to blend the chemotherapeutic approach with nanotechnology. Next section will discuss the recent development of nanotechnology-based drug delivery systems for overcoming drug resistance, therapy failure and drug withdrawn in degenerative diseases.

\section{Insulin delivery}

Recently diabetes mellitus has become epidemic and the incidence rate of this disease is rising rapidly. Though insulin administration is straightforward treatment of disease, the approach for insulin and other macromolecular diabetic therapies (glucagon-like peptide 1) is subcutaneous injection, which is painful and inconvenient, and leads to poor patient compliance. An innovative approach of oral insulin spray formulation has come up as an alternative to injectable insulin. The oral insulin is administered via buccal mucosal tract and is absorbed directly without any loss compared to the subcutaneous insulin injection. One of the major limitations of oral insulin delivery is low oral bioavailability of the hormone. Nanotechnology-based approach with the synthesis of polymeric nanoparticles and enteric coating has lessened the issue to some extent with limited success. It has been reported that nanosized negatively charged hydrophobic carrier for insulin has resulted in best oral absorption. $\beta$-Cyclodextrin and PLGA are considered as good candidates for coating material [235]. These polymers prevent degradation of insulin due to $\mathrm{pH}-$ dependent release in the gastrointestinal tract as well as self-aggregation of the hormone at neutral $\mathrm{pH}$ and provide stability to insulin [236]. AuNPs have been investigated as a good candidate for transmucosal insulin delivery. The biocompatible metal takes better control of blood glucose level in comparison to subcutaneous injection [237]. Another interesting approach is the fabrication of a nanocomposite membrane with glucose sensor which regulates the release of insulin. When the concentration of glucose is high, the $\mathrm{pH}$ level lowers down due to the oxidation of glucose to gluconic acid and this lowering of $\mathrm{pH}$ causes shrinkage of hydrogel membrane and releases insulin at faster rate [238].

\section{BBB crossed drug design}

The BBB is a primary hindrance towards drug development of brain and it has been regarded as one of the most important obstacle which limits the future growth of neurotherapeutics. The BBB is a highly sensitive neuronal organ formed by the specialized endothelial cells with tight junctions. It plays a key role in extruding the macromolecular neurotherapeutics from the brain [239]. Simultaneously, the BBB provides obstruction to potentially effective therapeutic agents with CNS disease. Several nanoparticles have been synthesized to enable delivery of therapeutic drugs across the BBB. Nanotechnology-based drug delivery vectors have the capability to reach the brain parenchyma evading these obstacles because they can be administered intracerebrally and can release their payload in a controlled manner. Circumvention of the physiological barriers depends on the morphology and physicochemical traits of the nanoparticles, which should be competent with the type of tissue or targeted cell.

The delivery of drug molecules across the BBB follows two basic perspective, molecular approach, and polymeric carrier approach. In the molecular approach, drugs are nanoionized or ligand attached and can be administered to target brain cells. The drugs can be further activated by enzymes afterwards inside the target cells. Yet due to the lack of availability of such customized drugs, only certain drugs with specific functionality can be targeted molecularly. Additionally, a metabolic pathway is always required for the activation of these drugs inside CNS-further narrowing options. Another approach of employing nanodrug to CNS is to deliver drug via polymeric carriers. These vectors can be tuned by choosing the matrix or polymer system and can be administered intrathecally, intravenously, or as an implantable cerebral device. Nanocarriers such as liposomes and polymeric nanoparticles are adept for protecting the payload drugs from being metabolized and ensure a sustained release of the embedded substances. For instance, Gliadel ${ }^{\circledR}$ is a wafer composed of polyanhydride polymer (poly [1, 3-bis(carb-oxyphenoxy) propane-co-sebacic acid]) (PPCP-SA) releases carmustine (BCNU) in a sustained manner post-brain implantation [240, 241]. 


\section{Active targeting agents to increase drug delivery systems for overcoming MDR}

\section{Aptamers}

Aptamers are single-stranded artificial oligonucleotide (DNA or RNA; 6-26 kDa) sequences, usually 20-60 bases long, that can fold into secondary and tertiary structures, with high affinity to bind a wide range of targets Fig. 6, including amino acids, drugs, proteins or even entire cells, with high specificity [242-246]. A breakthrough in the selection of cell-specific aptamers has provided redundancy in targeted drug delivery. Generally, aptamers are synthesized through an amplification and in vitro selection process known as the SELEX (systematic evolution of ligands by exponential enrichment). The selected aptamers have dissociation constants ranging from nanomolar to picomolar level. Till now, more than 200 aptamers have been isolated against a variety of biological targets, such as therapeutic targets, cell surface antigens, and growth factors like VEGF. The most significant one is the FDA approved aptamer against VEGF known as Pegaptanib, for the medication of age-related macular degeneration (AMD) [247]. Researchers have revolutionized biomedicine with the advent of aptamer-conjugated nanoparticles. These novel molecules have immense application including early diagnosis and drug delivery. For instance, the $2^{\prime}$-fluorinated A10 RNA aptamer-conjugated PLGA-PEG nanoparticles shall be adopted in targeted PSMA in case of prostate cancer model [248]. These PLGA-PEG-Apt nanoparticles can boost the therapeutic effect of anticancer drugs and lessen the systemic toxicity when compared to non-targeted nanoparticles. Lately, researchers have been working on DNA aptamers, generated through cell-SELEX, which have been conjugated with different types of nanoparticles (e.g., magnetic and AuNPs) for the early detection of cancer and its treatment [249-251]. At present, all nanoparticles using aptamers as target agents are going through preclinical phase.

\section{Nucleic acid delivery}

During the recent years, nucleic acid (DNA, miRNA, siRNA, etc.)-based nanoparticles are used as a potential tool in the modulation of drug resistance in tumors, effectively decreasing P-gp/MDR1 expression in vivo. The employed system uses RNA interference (RNAi) technology to selectively turn down the activity of oncogenes and deactivate some related molecular pathways. RNAi is a natural conserved mechanism in which RNA molecules inhibit gene expression, typically by inducing sequencespecific degradation of mRNA molecules in which siRNA molecules (about 20-25 base pairs long) are also involved. Recently, Meng et al. studied thoroughly the dual delivery of doxorubicin (Dox) and P-gp siRNA loaded in MSNPs. $\mathrm{He}$ found that the intracellular and intranuclear drug concentration has been reported to increase due to P-gp gene knockdown by siRNA [252]. In refractory metastatic melanoma transferrin receptors have been targeted with PEG-coated siRNA nanoparticles and observed that the nucleic acid-conjugated nanoparticle silenced target geneM2 ribonucleotide reductase [253]. Studies have
Fig. 6 Aptamer-based drug delivery system in cancer cell

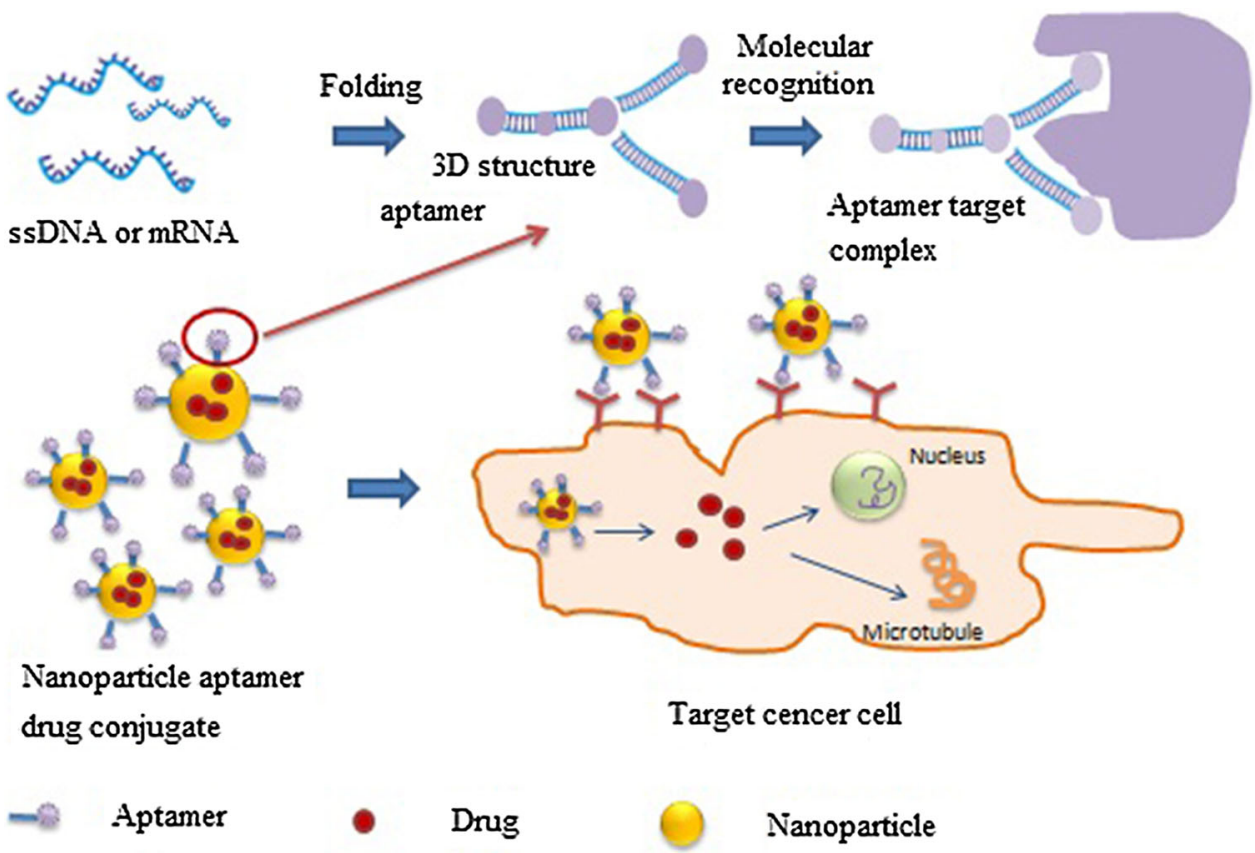


revealed that liposome-based cationic and anionic polycation-DNA nanoparticles loaded with C-Myc siRNA and Dox suppressed MDR1 gene expression via silencing the transcription level by intercalation of Dox, targeting transcription factors, topoisomerase II inhibition, and tumor regression.

\section{Receptor ligands (peptides) targeting agents}

One challenge to the use of targeting ligands for directing nanocarriers to overexpressed tumor cell receptors (or other epitopes) is the ability to selectively deliver therapeutic agents to these cells while sparing off-target (healthy) cells [254]. It is widely accepted that targeting ligands directing therapeutic agents (or, in the case of monoclonal antibodies, themselves acting as therapeutic agents) can be used to select between cells with vastly different receptor profiles for a given receptor. Peptides (10-15 amino acids) are able to bind target proteins, cells and tissues in a specific manner. Furthermore, they are characterized by reduced immunogenicity, high stability, and easy synthesis, scale-up, and chemical conjugations to nanocarriers. Phage display is emerging as the most popular approach, which has successfully isolated peptide ligands for somatostatin receptors, hormone receptors (LHRH receptors), and markers for the tumor vasculature. In addition to targeting cell surface receptors, effective peptide ligands also have been developed for extracellular matrix (ECM) receptors such as heparin sulphate and hyaluronan (HA), which are overexpressed in tumors. HA-targeted liposomes showed significantly improved circulation time and uptake by HA receptor-expressing tumors in vivo. The most common peptide used for targeting is RGD; binds integrins on the cell surface that affect cell migration, growth, differentiation and apoptosis in addition to cell-cell integrations. Integrin $\alpha_{v} \beta_{3}$ that binds to RGD peptides with high affinity is involved in intracellular signaling and direct roles in tumor angiogenesis [255]. Recent uses of RGD peptides for treating cancer include conjugation of PEGylated RGD peptides to gold nanoshells. These nanoparticles targeted U87 glioblastoma cells with a $267-n M$ IC $_{50}$ [256].

\section{pH-responsive nanoparticles for overcoming MDR}

The tumor microenvironment of cancer is one of the major areas to be focused for studying and designing a myriad of new therapy modality. For further exploration of this novel therapy, researchers have developed environmentally responsive nanoparticle for overcoming the drug resistance in tumor. This stimuli-responsive drug delivery system when exposed to external stimuli of tumor area produce physicochemical changes that favor drug release at the target site. The anticancer therapeutics can be released by an appropriate stimulus such as glucose, $\mathrm{pH}$, and temperature [257]. The nanocarriers for stimuli-responsive particles are engineered with specific material composition that can respond to the pathological "triggers" occurring in target site as the disease progresses. Among all applied stimuli such as $\mathrm{pH}$, ionic strength, redox potential, and enzymatic activities, $\mathrm{pH}$ gradient has been widely explored by the researchers for selective release of anticancer drugs as acidic $\mathrm{pH}$ as an internal stimulus has been considered as an ideal trigger. This is due to fact that the $\mathrm{pH}$ in the tumor microenvironment of both primary and metastasized tumors is lower than the $\mathrm{pH}$ of normal tissue. Such nanoparticles are formulated with those carrier materials that exhibit $\mathrm{pH}$-dependent swelling. Many review articles have introduced stimulation-sensitive polymers or their combinations with other materials in drug delivery. Till now various types of delivery systems, such as polymeric micelles, vesicles, liposomes, dendrimers, and hydrogels with stimulation-sensitive structures, have been manipulated to obtain desired stimulation-sensitive property. For instance, acrylate-based hydrophobic polymers with hydroxyl groups have been used for cross linking nanoparticles and were further coated with $\mathrm{pH}$-labile protecting groups. At neutral $\mathrm{pH}$ the NPs were found stable, but as the protecting group was cleaved and the hydroxyl groups were exposed to mildly acidic $\mathrm{pH}(\sim \mathrm{pH} 5)$, NPs swelled. This hydrophobic-to-hydrophilic transformation causes the swelling of subsequent drug release. Again, Hsin-Hung Chen et al. developed $\mathrm{pH}-$ responsive therapeutic cholesterol-PEG adduct-coated solid lipid nanoparticles (C-PEG-SLNs) which carried DOX capable of evading P-glycoprotein-mediated multidrug resistance (MDR) of breast cancer cell [258].

\section{Tumor drug delivery by EPR for overcoming MDR}

Targeted drug delivery to tumor sites is one of the crucial goals in drug delivery. Many solid tumors are known to develop a defective lymphatic drainage system, causing the molecules and nanoparticles to be retained there. The cutoff size of gap junctions between endothelial cells in permeable tumor blood vessels is around $200-800 \mathrm{~nm}$, which enables accumulation of nanoparticles. After accumulation in the tumor tissues, the drug is released into extracellular matrices, where it diffuses into intracellular sites.

For decades, conventional treatment for tumor is delivery of the drug systemically by intravenous injection. The greater the dose of drug, the more effectively it kills the malignant cells. Still, the dose to eradicate malignant 
cell completely is difficult to optimize. This is because it elevates toxicity level of the patient leading to increased mortality. When the solid tumor grows in size new vasculature formation occurs which is known as angiogenesis. Normal healthy tissues maintain proper cell barrier but malignant cells lose attachment of pericytes and results in leaky vasculature. In normal cell, the endothelial junction has a pore size of 6-7 nm which does not allow the delivery of particulate drugs but tumor microvessels are permeable to nanoparticles, liposomes up to $600 \mathrm{~nm}$. This delivery of macromolecule through leaky vasculature of tumor cells is called enhanced permeability retention. Macromolecules with molecular weight ranging from 45 to $75 \mathrm{kD}$ passively accumulate in the tumor cell but the penetration of drug into the microvasculature is gradually reduced. The advent of Nanocarrier drug allows transportation of the drug to the core of the cell. Again, the tumor cell expresses some specific receptor molecules which can be targeted via nanodrugs and antibodies. Liposomes and polymeric micelles entrap water-insoluble anticancer drugs such as tamoxifen, paclitaxel and transport to the target site. So the EPR effect coupled with active targeting with nanovectors can achieve a high payload of drug within the tumor tissue.

\section{Nanoparticles targeting cancer stem cells for overcoming MDR}

Cancer stem cells (CSCs) are more resistant to treatment of conventional chemotherapeutics and this drug resistance results in widespread metastasis during the late stages and eminent accumulation of CSCs. Anticancer drugs can often shrink tumors but cannot eradicate the CSCs. They cause the tumor re-growth or spread metastasis throughout the body [259]. CSC resistance is mainly achieved due to a series of events: increased $\mathrm{Wnt} / \beta$-catenin and Notch signaling, transformation of growth factor- $\beta$, hedgehog signaling, increased levels of ATP cassette reports, alteration in DNA repair mechanisms, specific markers (aldehyde dehydrogenases, CD44, CD90, and CD133) and slow proliferation rate [260]. Nanomedicine significantly offers a wide range of treatment therapies in comparison to existing anticancer drugs. Targeted nanosize drug delivery system such as liposome, nanoparticle, micelles, nucleic acid, nanogels can potentially overcome the shortcomings of available anticancer drugs owing to its high stability, specificity and highly controlled drug release. At present only a few drugs have been studied to have potential efficacy against CSCs. The researchers have demonstrated that nanoparticulate form of the anticancer drug doxorubicin encapsulated within chitosan, a natural polysaccharide can specifically target CSCs. Reaching the acidic $\mathrm{pH}$ in the tumor microenvironment, the nanoparticles degrade and release the drug. Tests performed on tiny, tissue-like clumps of both normal and cancer stem cells in vitro and on human breast tumors grown in mice showed that the therapy successfully killed CSCs and destroyed tumors. The mice showed no obvious side effects.

\section{Conclusion and future direction}

In the health systems more specifically on development of innovative health policies, nanomedicine has proved to be a promising area which has paved the way for new commercial solutions that will lead to benefits to individual, healthcare system and society in general. The application of nanotechnology in drug delivery system is increasing immensely due to its better control over the physical and biological characteristics of the drug compared to conventional therapeutic approaches. Extensive research is being conducted for better understanding of the degenerative disease pathways at molecular and cellular level. The success stories for preclinical approaches to clinical trial using nanoparticle-based approach for cancer therapy is still limited. The better fabrication of nanodrug for site specificity is one of the vital issues needed to be addressed. To tract the disease progression, invention of the molecular markers with nanoparticles may help in active tumor targeting. Moreover, intensive in vivo trials should be carried out for the evaluation of the pharmacokinetic and the biodistribution profile of the drug-loaded nanoparticles.

Nanotechnology applications have all the potential to revolutionize the drug delivery system in degenerative diseases. Biofabrication of the drug with careful and radical selection of the polymer is another factor to be considered. A simultaneous balance of development of nanotechnology-based therapeutic approach and safety of human healthcare is a matter of substantial concern as the field of nanotechnology is promising yet. To study the underlying mechanism of drug, diseases and translation of findings from bench to bedside with collaboration of scientists and clinicians are ultimate objectives of development of nanomedicine. The present review has demonstrated many different applications of nanotechnology-based drug delivery systems that are being used to fight against degenerative diseases.

Acknowledgements The authors are grateful to Chancellor, Vicechancellor and Directors of Kalasalingam University, Krishnankoil, India, and Department of Chemical Engineering, Jadavpur University, Kolkata, India, for research fellowships. We also thank to Prof. Z. Maciej Gliwicz, Ms. Ewa Babkiwics, and Dr. Piotr Maszczyk, Department of Hydrobiology, Faculty of Biology, University of Warsaw, Warsaw, Poland. 
Author contribution statement All the authors contributed equally to the preparation of this research article.

\section{Compliance with ethical standards}

Conflict of interest The authors declare no competing financial interest.

Open Access This article is distributed under the terms of the Creative Commons Attribution 4.0 International License (http://crea tivecommons.org/licenses/by/4.0/), which permits unrestricted use, distribution, and reproduction in any medium, provided you give appropriate credit to the original author(s) and the source, provide a link to the Creative Commons license, and indicate if changes were made.

\section{References}

1. Murray, C.J., Lopez, A.D.: Global and regional cause-of-death patterns in 1990. Bull. World Health Organ. 72(3), 447 (1994)

2. Finkel, T., Holbrook, N.J.: Oxidants, oxidative stress and the biology of ageing. Nature 408(6809), 239-247 (2000)

3. Cui, H., Kong, Y., Zhang, H.: Oxidative stress, mitochondrial dysfunction, and aging. J. Signal Trans. (2012)

4. Uttara, B., Singh, A.V., Zamboni, P., Mahajan, R.T.: Oxidative stress and neurodegenerative diseases: a review of upstream and downstream antioxidant therapeutic options. Curr. Neuropharmacol. 7(1), 65-74 (2009)

5. Alam, Q., ZubairAlam, M., Karim, S., H Gan, S., A Kamal, M., Jiman-Fatani, A., A Damanhouri, G., M Abuzenadah, A., G Chaudhary, A., Haque, A.: A nanotechnological approach to the management of Alzheimer disease and type 2 diabetes. CNS and Neurological Disorders-Drug Targets (Formerly Current Drug Targets-CNS \& Neurological Disorders) 13(3), 478-486 (2014)

6. Oesterling, B.M., Gulati, A., Joshi, M.D.: Nanocarrier-based approaches for treatment and detection of Alzheimer's disease. J. Nanosci. Nanotechnol. 14(1), 137-156 (2014)

7. Suri, S.S., Fenniri, H., Singh, B.: Nanotechnology-based drug delivery systems. J. Occupat. Med. Toxicol. 2(1), 16 (2007)

8. Davis, M.E., Zuckerman, J.E., Choi, C.H.J., Seligson, D., Tolcher, A., Alabi, C.A., Yen, Y., Heidel, J.D., Ribas, A.: Evidence of RNAi in humans from systemically administered siRNA via targeted nanoparticles. Nature 464(7291), 1067-1070 (2010)

9. Hrkach, J., Von Hoff, D., Ali, M.M., Andrianova, E., Auer, J., Campbell, T., De Witt, D., Figa, M., Figueiredo, M., Horhota, A.: Preclinical development and clinical translation of a PSMAtargeted docetaxel nanoparticle with a differentiated pharmacological profile. Science Translational Medicine 4(128), 128ra139-128ra139 (2012)

10. Al-Quadeib, B.T., Radwan, M.A., Siller, L., Horrocks, B., Wright, M.C.: Stealth Amphotericin B nanoparticles for oral drug delivery: in vitro optimization. Saudi Pharmac. J. 23(3), 290-302 (2015)

11. Avgoustakis, K., Beletsi, A., Panagi, Z., Klepetsanis, P., Karydas, A.G., Ithakissios, D.S.: PLGA-mPEG nanoparticles of cisplatin: in vitro nanoparticle degradation, in vitro drug release and in vivo drug residence in blood properties. J. Control. Release 79(1), 123-135 (2002)

12. Li, K., Yu, L., Liu, X., Chen, C., Chen, Q., Ding, J.: A longacting formulation of a polypeptide drug exenatide in treatment of diabetes using an injectable block copolymer hydrogel. Biomaterials 34(11), 2834-2842 (2013)

13. Masood, F., Chen, P., Yasin, T., Fatima, N., Hasan, F., Hameed, A.: Encapsulation of Ellipticine in poly-(3-hydroxybutyrate-co- 3-hydroxyvalerate) based nanoparticles and its in vitro application. Mater. Sci. Eng. C 33(3), 1054-1060 (2013)

14. Jain, A.K., Thanki, K., Jain, S.: Co-encapsulation of tamoxifen and quercetin in polymeric nanoparticles: implications on oral bioavailability, antitumor efficacy, and drug-induced toxicity. Mol. Pharm. 10(9), 3459-3474 (2013)

15. Khuroo, T., Verma, D., Talegaonkar, S., Padhi, S., Panda, A.K., Iqbal, Z.: Topotecan-tamoxifen duple PLGA polymeric nanoparticles: investigation of in vitro, in vivo and cellular uptake potential. Int. J. Pharm. 473(1), 384-394 (2014)

16. Suseela, P., Premkumar, K., Saraswathy, S.D.: Formulation, characterization and pharmacokinetic evaluation of naringeninloaded gastroretentive mucoadhesive polymeric nanosystem for oral drug delivery. J. Drug Deliv. Therap. 5(2), 107-114 (2015)

17. Salehi, P., Makhoul, G., Roy, R., Malhotra, M., Mood, Z.A., Daniel, S.J.: Curcumin loaded NIPAAM/VP/PEG-A nanoparticles: physicochemical and chemopreventive properties. J. Biomater. Sci. Polym. Ed. 24(5), 574-588 (2013)

18. Avachat, M.A., Oswal, M.Y., Gujar, N.K., Shah, D.R.: Preparation and characterization of rivastigmine loaded human serum albumin (HSA) nanoparticles. Curr. Drug Deliv. 11(3), 359-370 (2014)

19. Greish, K., Sawa, T., Fang, J., Akaike, T., Maeda, H.: SMAdoxorubicin, a new polymeric micellar drug for effective targeting to solid tumours. J. Control. Release 97(2), 219-230 (2004)

20. Tsuji, A., Hamaguchi, T., Yamaguchi, K., Takeda, K., Uetake, H., Esaki, T., Amagai, K., Sugimoto, N., Baba, H., Kimura, M.: A phase II study of NK012, a polymeric micelle formulation of $\mathrm{SN}-38$, in colorectal cancer patients who had received prior oxaliplatin-based regimen. In: ASCO Annual Meeting Proceedings. p. 3527 (2015)

21. Uchino, H., Matsumura, Y., Negishi, T., Koizumi, F., Hayashi, T., Honda, T., Nishiyama, N., Kataoka, K., Naito, S., Kakizoe, T.: Cisplatin-incorporating polymeric micelles (NC-6004) can reduce nephrotoxicity and neurotoxicity of cisplatin in rats. Br. J. Cancer 93(6), 678-687 (2005)

22. Musumeci, T., Ventura, C.A., Giannone, I., Ruozi, B., Montenegro, L., Pignatello, R., Puglisi, G.: PLA/PLGA nanoparticles for sustained release of docetaxel. Int. J. Pharm. 325(1), 172-179 (2006)

23. Dignam, J.D., Qu, X., Ren, J., Chaires, J.B.: Daunomycin binding to detergent micelles: a model system for evaluating the hydrophobic contribution to drug-DNA interactions. J. Phys. Chem. B 111(39), 11576-11584 (2007)

24. Yang, D., Van, S., Jiang, X., Yu, L.: Novel free paclitaxelloaded poly ( $\mathrm{L}-\beta$-glutamylglutamine)-paclitaxel nanoparticles. Int. J. Nanomed. 6, 85-91 (2011)

25. Veronese, F.M., Schiavon, O., Pasut, G., Mendichi, R., Andersson, L., Tsirk, A., Ford, J., Wu, G., Kneller, S., Davies, J.: PEGdoxorubicin conjugates: influence of polymer structure on drug release, in vitro cytotoxicity, biodistribution, and antitumor activity. Bioconjug. Chem. 16(4), 775-784 (2005)

26. Singer, J.W., Baker, B., de Vries, P., Kumar, A., Shaffer, S., Vawter, E., Bolton, M., Garzone, P.: Poly-(1)-glutamic acidpaclitaxel (CT-2103)[XYOTAX], a biodegradable polymeric drug conjugate. In: Polymer drugs in the clinical stage. pp. 81-99. Springer (2004)

27. Lodha, A., Lodha, M., Patel, A., Chaudhuri, J., Dalal, J., Edwards, M., Douroumis, D.: Synthesis of mesoporous silica nanoparticles and drug loading of poorly water soluble drug cyclosporin A. J. Pharm. Bioal. Sci. 4(Suppl 1), S92 (2012)

28. Hom, C., Lu, J., Liong, M., Luo, H., Li, Z., Zink, J.I., Tamanoi, F.: Mesoporous silica nanoparticles facilitate delivery of siRNA to shutdown signaling pathways in mammalian cells. Small 6(11), 1185-1190 (2010) 
29. Atabaev, T.S., Urmanova, G., Hong, N.H.: Highly mesoporous silica nanoparticles for potential drug delivery applications. Nano Life 4(03), 1441003 (2014)

30. Quan, G., Pan, X., Wang, Z., Wu, Q., Li, G., Dian, L., Chen, B., $\mathrm{Wu}, \mathrm{C}$. : Lactosaminated mesoporous silica nanoparticles for asialoglycoprotein receptor targeted anticancer drug delivery. J. Nanobiotechnol. 13(1), 7 (2015)

31. Hsiao, M.-H., Larsson, M., Larsson, A., Evenbratt, H., Chen, Y.Y., Chen, Y.-Y., Liu, D.-M.: Design and characterization of a novel amphiphilic chitosan nanocapsule-based thermo-gelling biogel with sustained in vivo release of the hydrophilic antiepilepsy drug ethosuximide. J. Control. Release 161(3), 942-948 (2012)

32. Tummala, S., Kumar, M.N.S., Prakash, A.: Formulation and characterization of 5-fluorouracil enteric coated nanoparticles for sustained and localized release in treating colorectal cancer. Saudi Pharm. J. 23(3), 308-314 (2015)

33. Amorim, C.d.M., Couto, A.l.G., Netz, D.J.A., de Freitas, R.A., Bresolin, T.M.B.: Antioxidant idebenone-loaded nanoparticles based on chitosan and $\mathrm{N}$-carboxymethylchitosan. Nanomedicine: Nanotechnology, Biology and Medicine 6(6), 745-752 (2010)

34. Xu, R., Fisher, M., Juliano, R.L.: Targeted albumin-based nanoparticles for delivery of amphipathic drugs. Bioconjug. Chem. 22(5), 870-878 (2011)

35. Wong, T.W., Sumiran, N.: Oral calcium pectinate insulin nanoparticles: influences of alginate, sodium chloride and Tween 80 on their blood glucose lowering performance. J. Pharm. Pharmacol. 66(5), 646-657 (2014)

36. Das, S., Jagan, L., Isiah, R., Rajesh, B., Backianathan, S., Subhashini, J.: Nanotechnology in oncology: characterization and in vitro release kinetics of cisplatin-loaded albumin nanoparticles: Implications in anticancer drug delivery. Indian J. Pharmacol. 43(4), 409 (2011)

37. Xiao, Y., Liu, Y., Yang, S., Zhang, B., Wang, T., Jiang, D., Zhang, J., Yu, D., Zhang, N.: Sorafenib and Gadolinium coloaded liposomes for drug delivery and MRI-guided HCC treatment. Colloids and Surfaces B: Biointerfaces (2016)

38. Numico, G., Castiglione, F., Granetto, C., Garrone, O., Mariani, G., Di Costanzo, G., La Ciura, P., Gasco, M., Ostellino, O., Porcile, G.: Single-agent pegylated liposomal doxorubicin $\left(\right.$ Caelix $\left.{ }^{\circledR}\right)$ in chemotherapy pretreated non-small cell lung cancer patients: a pilot trial. Lung Cancer 35(1), 59-64 (2002)

39. Kunjiappan, S., Chowdhury, R., Bhattacharjee, C.: A green chemistry approach for the synthesis and characterization of bioactive gold nanoparticles using Azolla microphylla methanol extract. Front. Mater. Sci. 8(2), 123-135 (2014)

40. Narayani, R., Rao, K.P.: Biodegradable microspheres using two different gelatin drug conjugates for the controlled delivery of methotrexate. Int. J. Pharm. 128(1), 261-268 (1996)

41. Chowdhury, A., Kunjiappan, S., Bhattacharjee, C., Chowdhury, R.: Green synthesis and characterization of biocompatible silver nanoparticles using Brassica oleracea L. leaf extract. Int. J. Curr. Res. 6(4), 6166-6174 (2014)

42. Dugan, L.L., Turetsky, D.M., Du, C., Lobner, D., Wheeler, M., Almli, C.R., Shen, C.K.F., Luh, T.-Y., Choi, D.W., Lin, T.-S.: Carboxyfullerenes as neuroprotective agents. Proc. Natl. Acad. Sci. 94(17), 9434-9439 (1997)

43. Alibolandi, M., Alabdollah, F., Sadeghi, F., Mohammadi, M., Abnous, K., Ramezani, M., Hadizadeh, F.: Dextran-b-poly (lactide-co-glycolide) polymersome for oral delivery of insulin: in vitro and in vivo evaluation. J. Control. Release 227, 58-70 (2016)

44. Zhang, R.X., Cai, P., Zhang, T., Chen, K., Li, J., Cheng, J., Pang, K.S., Adissu, H.A., Rauth, A.M., Wu, X.Y.: Polymer-lipid hybrid nanoparticles synchronize pharmacokinetics of co- encapsulated doxorubicin-mitomycin $\mathrm{C}$ and enable their spatiotemporal co-delivery and local bioavailability in breast tumor. Nanomed. Nanotechnol. Biol. Med. 12(5), 1279-1290 (2016)

45. Uchegbu, I.F., Double, J.A., Kelland, L.R., Turton, J.A., Florence, A.T.: The activity of doxorubicin niosomes against an ovarian cancer cell line and three in vivo mouse tumour models. J. Drug Target. 3(5), 399-409 (1996)

46. Rogerson, A., Cummings, J., Willmott, N., Florence, A.T.: The distribution of doxorubicin in mice following administration in niosomes. J. Pharm. Pharmacol. 40(5), 337-342 (1988)

47. Liu, Z., Chen, K., Davis, C., Sherlock, S., Cao, Q., Chen, X., Dai, H.: Drug delivery with carbon nanotubes for in vivo cancer treatment. Can. Res. 68(16), 6652-6660 (2008)

48. Ferrari, M.: Cancer nanotechnology: opportunities and challenges. Nat. Rev. Cancer 5(3), 161-171 (2005)

49. Thakor, A.S., Gambhir, S.S.: Nanooncology: the future of cancer diagnosis and therapy. CA Cancer J. Clin. 63(6), 395-418 (2013)

50. Ochekpe, N.A., Olorunfemi, P.O., Ngwuluka, N.C.: Nanotechnology and drug delivery part 1: background and applications. Tropical Journal of Pharmaceutical Research 8(3) (2009)

51. Singh, R., Lillard, J.W.: Nanoparticle-based targeted drug delivery. Exp. Mol. Pathol. 86(3), 215-223 (2009)

52. Wilson, B., Samanta, M.K., Santhi, K., Kumar, K.P.S., Paramakrishnan, N., Suresh, B.: Targeted delivery of tacrine into the brain with polysorbate 80 -coated poly (n-butylcyanoacrylate) nanoparticles. Eur. J. Pharm. Biopharm. 70(1), 75-84 (2008)

53. Wilson, B., Samanta, M.K., Santhi, K., Kumar, K.P.S., Paramakrishnan, N., Suresh, B.: Poly (n-butylcyanoacrylate) nanoparticles coated with polysorbate 80 for the targeted delivery of rivastigmine into the brain to treat Alzheimer's disease. Brain Res. 1200, 159-168 (2008)

54. Lazar, A.N., Mourtas, S., Youssef, I., Parizot, C., Dauphin, A.L., Delatour, B.T., Antimisiaris, S.G., Duyckaerts, C.: Curcuminconjugated nanoliposomes with high affinity for $A \beta$ deposits: possible applications to Alzheimer disease. Nanomed. Nanotechnol. Biol. Med. 9(5), 712-721 (2013)

55. Huang, X., El-Sayed, M.A.: Gold nanoparticles: optical properties and implementations in cancer diagnosis and photothermal therapy. J. Adv. Res. 1(1), 13-28 (2010)

56. Arvizo, R.R., Bhattacharyya, S., Kudgus, R.A., Giri, K., Bhattacharya, R., Mukherjee, P.: Intrinsic therapeutic applications of noble metal nanoparticles: past, present and future. Chem. Soc. Rev. 41(7), 2943-2970 (2012)

57. Thakkar, K.N., Mhatre, S.S., Parikh, R.Y.: Biological synthesis of metallic nanoparticles. Nanomed. Nanotechnol. Biol. Med. 6(2), 257-262 (2010)

58. Elvira Nuez-Anita, R., Susana Acosta-Torres, L., Vilar-Pineda, J., Carlos Martinez-Espinosa, J., de la Fuente-Hernandez, J., Manuel Castano, V.: Toxicology of antimicrobial nanoparticles for prosthetic devices. Int. J. Nanomed. 9, 3999-4006 (2014)

59. Lokina, S., Narayanan, V.: Antimicrobial and anticancer activity of gold nanoparticles synthesized from grapes fruit extract. Chem. Sci. Trans. 2(S1), S105-S110 (2013)

60. Yeo, S.Y., Lee, H.J., Jeong, S.H.: Preparation of nanocomposite fibers for permanent antibacterial effect. J. Mater. Sci. 38(10), 2143-2147 (2003)

61. Zhang, J.-P., Chen, P., Sun, C.-H., Hu, X.-J.: Sonochemical synthesis of colloidal silver catalysts for reduction of complexing silver in DTR system. Appl. Catal. A 266(1), 49-54 (2004)

62. Zhang, W., Qiao, X., Chen, J., Wang, H.: Preparation of silver nanoparticles in water-in-oil AOT reverse micelles. J. Colloid Interf. Sci. 302(1), 370-373 (2006)

63. Chimentao, R.J., Kirm, I., Medina, F., Rodriguez, X., Cesteros, Y., Salagre, P., Sueiras, J.E.: Different morphologies of silver 
nanoparticles as catalysts for the selective oxidation of styrene in the gas phase. Chem. Commun. 7, 846-847 (2004)

64. He, B., Tan, J.J., Liew, K.Y., Liu, H.: Synthesis of size controlled Ag nanoparticles. J. Mol. Catal. A: Chem. 221(1), 121-126 (2004)

65. Yu, D.-G.: Formation of colloidal silver nanoparticles stabilized by Na-poly ( $\gamma$-glutamic acid)-silver nitrate complex via chemical reduction process. Colloids Surf. B 59(2), 171-178 (2007)

66. Liu, Y.-C., Lin, L.-H.: New pathway for the synthesis of ultrafine silver nanoparticles from bulk silver substrates in aqueous solutions by sonoelectrochemical methods. Electrochem. Commun. 6(11), 1163-1168 (2004)

67. Mallick, K., Witcomb, M.J., Scurrell, M.S.: Self-assembly of silver nanoparticles in a polymer solvent: formation of a nanochain through nanoscale soldering. Mater. Chem. Phys. 90(2), 221-224 (2005)

68. Khomutov, G.B., Gubin, S.P.: Interfacial synthesis of noble metal nanoparticles. Mater. Sci. Eng., C 22(2), 141-146 (2002)

69. Oliveira, M.M., Ugarte, D., Zanchet, D., Zarbin, A.J.G.: Influence of synthetic parameters on the size, structure, and stability of dodecanethiol-stabilized silver nanoparticles. J. Colloid Interface Sci. 292(2), 429-435 (2005)

70. Egorova, E.M., Revina, A.A.: Synthesis of metallic nanoparticles in reverse micelles in the presence of quercetin. Colloids Surf. A 168(1), 87-96 (2000)

71. Song, J.Y., Kim, B.S.: Rapid biological synthesis of silver nanoparticles using plant leaf extracts. Bioprocess Biosyst. Eng. 32(1), 79-84 (2009)

72. Nune, S.K., Chanda, N., Shukla, R., Katti, K., Kulkarni, R.R., Thilakavathy, S., Mekapothula, S., Kannan, R., Katti, K.V.: Green nanotechnology from tea: phytochemicals in tea as building blocks for production of biocompatible gold nanoparticles. J. Mater. Chem. 19(19), 2912-2920 (2009)

73. Giljohann, D.A., Seferos, D.S., Daniel, W.L., Massich, M.D., Patel, P.C., Mirkin, C.A.: Gold nanoparticles for biology and medicine. Angew. Chem. Int. Ed. 49(19), 3280-3294 (2010)

74. Das, S.K., Das, A.R., Guha, A.K.: Synthesis of gold nanoparticles: a green chemical approach. In: International conference on soft system ICSS-2008, Kolkata, India, pp. 3-15 (2008)

75. Sperling, R.A., Gil, P.R., Zhang, F., Zanella, M., Parak, W.J.: Biological applications of gold nanoparticles. Chem. Soc. Rev. 37(9), 1896-1908 (2008)

76. Chen, Y.-P., Dai, Z.-H., Liu, P.-C., Chuu, J.-J., Lee, K.-Y., Lee, S.-L., Chen, Y.-J.: Effects of nanogold on the alleviation of carbon tetrachloride-induced hepatic injury in rats. Chin. J. Physiol. 55(5), 331-336 (2012)

77. Mukherjee, P., Bhattacharya, R., Wang, P., Wang, L., Basu, S., Nagy, J.A., Atala, A., Mukhopadhyay, D., Soker, S.: Antiangiogenic properties of gold nanoparticles. Clin. Cancer Res. 11(9), 3530-3534 (2005)

78. Gao, W., Xu, K., Ji, L., Tang, B.: Effect of gold nanoparticles on glutathione depletion-induced hydrogen peroxide generation and apoptosis in HL7702 cells. Toxicol. Lett. 205(1), 86-95 (2011)

79. Ghosh, R., Singh, L.C., Shohet, J.M., Gunaratne, P.H.: A gold nanoparticle platform for the delivery of functional microRNAs into cancer cells. Biomaterials 34(3), 807-816 (2013)

80. Markman, J.L., Rekechenetskiy, A., Holler, E., Ljubimova, J.Y.: Nanomedicine therapeutic approaches to overcome cancer drug resistance. Adv. Drug Deliv. Rev. 65(13), 1866-1879 (2013)

81. Webster, D.M., Sundaram, P., Byrne, M.E.: Injectable nanomaterials for drug delivery: carriers, targeting moieties, and therapeutics. Eur. J. Pharm. Biopharm. 84(1), 1-20 (2013)

82. Visaria, R.K., Griffin, R.J., Williams, B.W., Ebbini, E.S., Paciotti, G.F., Song, C.W., Bischof, J.C.: Enhancement of tumor thermal therapy using gold nanoparticle assisted tumor necrosis factor- $\alpha$ delivery. Mol. Cancer Ther. 5(4), 1014-1020 (2006)
83. Lee, J.-M., Yoon, T.-J., Cho, Y.-S.: Recent developments in nanoparticle-based siRNA delivery for cancer therapy. BioMed Res. Int. 2013 (2013)

84. Bonoiu, A.C., Mahajan, S.D., Ding, H., Roy, I., Yong, K.-T., Kumar, R., Hu, R., Bergey, E.J., Schwartz, S.A., Prasad, P.N.: Nanotechnology approach for drug addiction therapy: gene silencing using delivery of gold nanorod-siRNA nanoplex in dopaminergic neurons. Proc. Natl. Acad. Sci. 106(14), 5546-5550 (2009)

85. Cognet, L., Tardin, C., Boyer, D., Choquet, D., Tamarat, P., Lounis, B.: Single metallic nanoparticle imaging for protein detection in cells. Proc. Natl. Acad. Sci. 100(20), 11350-11355 (2003)

86. O’Neal, D.P., Hirsch, L.R., Halas, N.J., Payne, J.D., West, J.L.: Photo-thermal tumor ablation in mice using near infrared-absorbing nanoparticles. Cancer Lett. 209(2), 171-176 (2004)

87. Huang, J., Li, Q., Sun, D., Lu, Y., Su, Y., Yang, X., Wang, H., Wang, Y., Shao, W., He, N.: Biosynthesis of silver and gold nanoparticles by novel sundried Cinnamomum camphora leaf. Nanotechnology 18(10), 105104 (2007)

88. Lee, J., Kim, H.Y., Zhou, H., Hwang, S., Koh, K., Han, D.-W., Lee, J.: Green synthesis of phytochemical-stabilized $\mathrm{Au}$ nanoparticles under ambient conditions and their biocompatibility and antioxidative activity. J. Mater. Chem. 21(35), 13316-13326 (2011)

89. Jain, D., Daima, H.K., Kachhwaha, S., Kothari, S.L.: Synthesis of plant-mediated silver nanoparticles using papaya fruit extract and evaluation of their anti microbial activities. Digest $\mathrm{J}$. Nanomater. Biostruct. 4(3), 557-563 (2009)

90. Kim, J.S., Kuk, E., Yu, K.N., Kim, J.-H., Park, S.J., Lee, H.J., Kim, S.H., Park, Y.K., Park, Y.H., Hwang, C.-Y.: Antimicrobial effects of silver nanoparticles. Nanomed. Nanotechnol. Biol. Med. 3(1), 95-101 (2007)

91. Nadworny, P.L., Wang, J., Tredget, E.E., Burrell, R.E.: Antiinflammatory activity of nanocrystalline silver in a porcine contact dermatitis model. Nanomed. Nanotechnol. Biol. Med. 4(3), 241-251 (2008)

92. Rao, P.V., Nallappan, D., Madhavi, K., Rahman, S., Jun Wei, L., Gan, S.H.: Phytochemicals and Biogenic Metallic Nanoparticles as Anticancer Agents. Oxidative Medicine and Cellular Longevity 2016 (2016)

93. Ahamed, M., AlSalhi, M.S., Siddiqui, M.K.J.: Silver nanoparticle applications and human health. Clin. Chim. Acta 411(23-24), 1841-1848 (2010). doi:10.1016/j.cca.2010.08.016

94. Senyei, A., Widder, K., Czerlinski, G.: Magnetic guidance of drug carrying microspheres. J. Appl. Phys. 49(6), 3578-3583 (1978)

95. Widder, K.J., Senyei, A.E., Scarpelli, D.G.: Magnetic microspheres: a model system for site specific drug delivery in vivo. Exp. Biol. Med. 158(2), 141-146 (1978)

96. Grancharov, S.G., Zeng, H., Sun, S., Wang, S.X., O’Brien, S., Murray, C.B., Kirtley, J.R., Held, G.A.: Bio-functionalization of monodisperse magnetic nanoparticles and their use as biomolecular labels in a magnetic tunnel junction based sensor. J. Phys. Chem. B 109(26), 13030-13035 (2005)

97. McBain, S.C., Yiu, H.H.P., Dobson, J.: Magnetic nanoparticles for gene and drug delivery. Int. J. Nanomed. 3(2), 169 (2008)

98. Sun, X.: Mesoporous silica nanoparticles for applications in drug delivery and catalysis. Graduate Theses and Dissertations, Paper 12812. (2012)

99. Tang, F., Li, L., Chen, D.: Mesoporous silica nanoparticles: synthesis, biocompatibility and drug delivery. Adv. Mater. 24(12), 1504-1534 (2012)

100. Huh, S., Wiench, J.W., Trewyn, B.G., Song, S., Pruski, M., Lin, V.S.Y.: Tuning of particle morphology and pore properties in mesoporous silicas with multiple organic functional groups. Chem. Commun. 18, 2364-2365 (2003) 
101. Guo, H., Qian, H., Sun, S., Sun, D., Yin, H., Cai, X., Liu, Z., Wu, J., Jiang, T., Liu, X.: Hollow mesoporous silica nanoparticles for intracellular delivery of fluorescent dye. Chem. Cent. J. 5(1), 1 (2011)

102. Upadhyay, R.K.: Drug delivery systems, CNS protection, and the blood brain barrier. BioMed Res. Int. 2014 (2014)

103. Brooks, D.J.: Optimizing levodopa therapy for Parkinson's disease with levodopa/carbidopa/entacapone: implications from a clinical and patient perspective. Neuropsychiat. Dis. Treat. 4(1), 39 (2008)

104. Trapani, A., De Giglio, E., Cafagna, D., Denora, N., Agrimi, G., Cassano, T., Gaetani, S., Cuomo, V., Trapani, G.: Characterization and evaluation of chitosan nanoparticles for dopamine brain delivery. Int. J. Pharm. 419(1), 296-307 (2011)

105. Yang, X., Chen, Y., Hong, X., Wu, N., Song, L., Yuan, W., Liu, Z.: Levodopa/benserazide microspheres reduced levodopa-induced dyskinesia by downregulating phosphorylated GluR1 expression in 6-OHDA-lesioned rats. Drug Design Dev. Therapy 6, 341 (2012)

106. Shenoy, D.B., Amiji, M.M.: Poly (ethylene oxide)-modified poly (epsilon-caprolactone) nanoparticles for targeted delivery of tamoxifen in breast cancer. Int. J. Pharm. 293(1), 261-270 (2005)

107. Makeshwar, K.B., Wasankar, S.R.: Niosome: a novel drug delivery system. Asian J. Pharm. Res. 3(1), 16-20 (2013)

108. Moghassemi, S., Hadjizadeh, A.: Nano-niosomes as nanoscale drug delivery systems: an illustrated review. J. Control. Release 185, 22-36 (2014)

109. Junginger, H.E., Hofland, H.E.J., Bouwstra, J.A.: Liposomes and niosomes: interactions with human skin. Cosmet. Toilet 106(8), 45-50 (1991)

110. Kumar, B., Dwivedi, C., Tiwari, S.P., Satapathy, T.: Int. J. Res. Pharmacol. Pharmacother. (2014)

111. Liechty, W.B., Kryscio, D.R., Slaughter, B.V., Peppas, N.A.: Polymers for drug delivery systems. Annual Rev. Chem. Biomol. Eng. 1, 149 (2010)

112. Yurkovetskiy, A.V., Fram, R.J.: XMT-1001, a novel polymeric camptothecin pro-drug in clinical development for patients with advanced cancer. Adv. Drug Deliv. Rev. 61(13), 1193-1202 (2009)

113. Duncan, R.: Polymer conjugates as anticancer nanomedicines. Nat. Rev. Cancer 6(9), 688-701 (2006)

114. Lee, J.S., Park, K.D.: Biodegradable polymer vesicles: design and performance as drug delivery carriers. Biomater. Res. 15, $152(2011)$

115. Ahmed, F., Pakunlu, R.I., Brannan, A., Bates, F., Minko, T., Discher, D.E.: Biodegradable polymersomes loaded with both paclitaxel and doxorubicin permeate and shrink tumors, inducing apoptosis in proportion to accumulated drug. J. Control. Release 116(2), 150-158 (2006)

116. Li, S., Byrne, B., Welsh, J., Palmer, A.F.: Self-assembled Poly (butadiene)-b-poly (ethylene oxide) polymersomes as paclitaxel carriers. Biotechnol. Prog. 23(1), 278-285 (2007)

117. Chen, W., Meng, F., Cheng, R., Zhong, Z.: pH-Sensitive degradable polymersomes for triggered release of anticancer drugs: a comparative study with micelles. J. Control. Release 142(1), 40-46 (2010)

118. Madaan, K., Kumar, S., Poonia, N., Lather, V., Pandita, D.: Dendrimers in drug delivery and targeting: drug-dendrimer interactions and toxicity issues. J. Pharm. Bioall. Sci. 6(3), 139 (2014)

119. Tomalia, D.A., Baker, H., Dewald, J., Hall, M., Kallos, G., Martin, S., Roeck, J., Ryder, J., Smith, P.: A new class of polymers: starburst-dendritic macromolecules. Polym. J. 34(5), 132-147 (2002)
120. Dufes, C., Uchegbu, I., Schatzlein, A.G.: Dendrimers in gene delivery. Adv. Drug Deliv. Rev. 57(15), 2177-2202 (2005)

121. Kim, S., Shi, Y., Kim, J.Y., Park, K., Cheng, J.-X.: Overcoming the barriers in micellar drug delivery: loading efficiency, in vivo stability, and micelle-cell interaction. Expert Opinion on Drug Delivery 7(1), 49-62 (2010)

122. Gaucher, G.V., Dufresne, M.-H.L.N., Sant, V.P., Kang, N., Maysinger, D., Leroux, J.-C.: Block copolymer micelles: preparation, characterization and application in drug delivery. J. Control. Release 109(1), 169-188 (2005)

123. Croy, S., Kwon, G.: Polymeric micelles for drug delivery. Curr. Pharm. Des. 12(36), 4669-4684 (2006)

124. Bangham, A.D., Horne, R.W.: Negative staining of phospholipids and their structural modification by surface-active agents as observed in the electron microscope. J. Mol. Biol. 8(5), 660IN662-668IN610 (1964)

125. Spuch, C., Navarro, C.: Liposomes for targeted delivery of active agents against neurodegenerative diseases (Alzheimer's Disease and Parkinson's Disease). J. Drug Deliv. 2011 (2011)

126. Samad, A., Sultana, Y., Aqil, M.: Liposomal drug delivery systems: an update review. Curr. Drug Deliv. 4(4), 297-305 (2007)

127. Alexis, F., Pridgen, E.M., Langer, R., Farokhzad, O.C.: Nanoparticle technologies for cancer therapy. In: Drug delivery. pp. 55-86. Springer (2010)

128. Bagalkot, V., Badgeley, M.A., Kampfrath, T., Deiuliis, J.A., Rajagopalan, S., Maiseyeu, A.: Hybrid nanoparticles improve targeting to inflammatory macrophages through phagocytic signals. J. Control. Release 217, 243-255 (2015). doi:10.1016/j. jconrel.2015.09.027

129. Ge, J., Neofytou, E., Lei, J., Beygui, R.E., Zare, R.N.: Proteinpolymer hybrid nanoparticles for drug delivery. Small 8(23), 3573-3578 (2012)

130. Lohcharoenkal, W., Wang, L., Chen, Y.C., Rojanasakul, Y.: Protein nanoparticles as drug delivery carriers for cancer therapy. BioMed Research International 2014 (2014)

131. Elsadek, B., Kratz, F.: Impact of albumin on drug delivery-new applications on the horizon. J. Control. Release 157(1), 4-28 (2012)

132. Yousefpour, P., Chilkoti, A.: Co-opting biology to deliver drugs. Biotechnol. Bioeng. 111(9), 1699-1716 (2014)

133. Moghimi, S.M., Hunter, A.C., Murray, J.C.: Long-circulating and target-specific nanoparticles: theory to practice. Pharmacol. Rev. 53(2), 283-318 (2001)

134. Tokuda, Y.: Antibodies as molecular target-based therapy: trastuzumab. Int. J. Clin. Oncol. 8(4), 224-229 (2003)

135. Reddy, K.R.: Controlled-release, pegylation, liposomal formulations: new mechanisms in the delivery of injectable drugs. Ann. Pharmacother. 34(7-8), 915-923 (2000)

136. Elzoghby, A.O., Samy, W.M., Elgindy, N.A.: Albumin-based nanoparticles as potential controlled release drug delivery systems. J. Control. Release 157(2), 168-182 (2012)

137. Young Yhee, J., Lee, J., Chang, H., Lee, J., Chan Kwon, I., Kim, K.: Molecular imaging and targeted drug delivery using albumin-based nanoparticles. Curr. Pharm. Des. 21(14), 1889-1898 (2015)

138. Kratz, F.: Albumin as a drug carrier: design of prodrugs, drug conjugates and nanoparticles. J. Control. Release 132(3), 171-183 (2008)

139. Elzoghby, A.O.: Gelatin-based nanoparticles as drug and gene delivery systems: reviewing three decades of research. J. Control. Release 172(3), 1075-1091 (2013)

140. Chandy, T., Sharma, C.P.: Resorbable chitosan matrix-a promising biomaterial for the future. In: Biomedical Engineering Conference, 1995., Proceedings of the 1995 Fourteenth Southern 1995, pp. 282-285. IEEE 
141. Dhillon, G.S., Kaur, S., Brar, S.K., Verma, M.: Green synthesis approach: extraction of chitosan from fungus mycelia. Crit. Rev. Biotechnol. 33(4), 379-403 (2013)

142. Chaudhury, A., Das, S.: Recent advancement of chitosan-based nanoparticles for oral controlled delivery of insulin and other therapeutic agents. AAPS Pharm. Sci. Tech. 12(1), 10-20 (2011)

143. Harris, R., Lecumberri, E., Mateos-Aparicio, I., MengÃbar, M., Heras, A.: Chitosan nanoparticles and microspheres for the encapsulation of natural antioxidants extracted from Ilex paraguariensis. Carbohyd. Polym. 84(2), 803-806 (2011)

144. Raffa, V., Vittorio, O., Riggio, C., Ciofani, G., Cuschieri, A.: Physical properties of carbon nanotubes for therapeutic applications. In: Carbon Nanotubes for Biomedical Applications. pp. 3-26. Springer, (2011)

145. Zhang, W., Zhang, Z., Zhang, Y.: The application of carbon nanotubes in target drug delivery systems for cancer therapies. Nanoscale Res. Lett. 6(1), 1-22 (2011)

146. Ferluga, J., Kishore, U., Sim, R.B.: A potential anti-coagulant role of complement factor H. Mol. Immunol. 59(2), 188-193 (2014)

147. Jiang, W., Kim, B.Y.S., Rutka, J.T., Chan, W.C.W.: Advances and challenges of nanotechnology-based drug delivery systems. Expert Opin. Drug Deliv. 4(6), 621-633 (2007)

148. Chan, W.C.W.: Bio-applications of Nanoparticles, vol. 620. Springer Science and Business Media (2009)

149. Bakry, R., Vallant, R.M., Najam-ul-Haq, M., Rainer, M., Szabo, Z., Huck, C.W., Bonn, G.N.K.: Medicinal applications of fullerenes. Int. J. Nanomed. 2(4), 639 (2007)

150. Misra, R., Upadhyay, M., Mohanty, S.: Design considerations for chemotherapeutic drug nanocarriers. Pharmaceutica Analytica Acta 2014 (2014)

151. Liu, Y., Wang, W., Yang, J., Zhou, C., Sun, J.: pH-sensitive polymeric micelles triggered drug release for extracellular and intracellular drug targeting delivery. Asian J. Pharm. Sci. 8(3), 159-167 (2013)

152. Nguyen, K.T.: Targeted nanoparticles for cancer therapy: promises and challenges. J. Nanomed. Nanotechnol. 2011 (2011)

153. Cao, Z., Tong, R., Mishra, A., Xu, W., Wong, G.C.L., Cheng, J., $\mathrm{Lu}$, Y.: Reversible cell-specific drug delivery with aptamerfunctionalized liposomes. Angew. Chem. Int. Ed. 48(35), 6494-6498 (2009)

154. Muntimadugu, E., Kumar, R., Saladi, S., Rafeeqi, T.A., Khan, W.: CD44 targeted chemotherapy for co-eradication of breast cancer stem cells and cancer cells using polymeric nanoparticles of salinomycin and paclitaxel. Colloids Surf. B 143, 532-546 (2016)

155. Nagesh, P.K.B., Johnson, N.R., Boya, V.K.N., Chowdhury, P., Othman, S.F., Khalilzad-Sharghi, V., Hafeez, B.B., Ganju, A., Khan, S., Behrman, S.W.: PSMA targeted docetaxel-loaded superparamagnetic iron oxide nanoparticles for prostate cancer. Colloids Surf. B 144, 8-20 (2016)

156. Di-Wen, S., Pan, G.-Z., Hao, L., Zhang, J., Xue, Q.-Z., Wang, P., Yuan, Q.-Z.: Improved antitumor activity of epirubicin-loaded CXCR4-targeted polymeric nanoparticles in liver cancers. Int. J. Pharm. 500(1), 54-61 (2016)

157. Xie, X., Li, F., Zhang, H., Lu, Y., Lian, S., Lin, H., Gao, Y., Jia, L.: EpCAM aptamer-functionalized mesoporous silica nanoparticles for efficient colon cancer cell-targeted drug delivery. Eur. J. Pharm. Sci. 83, 28-35 (2016)

158. Singh, A., Xu, J., Mattheolabakis, G., Amiji, M.: EGFR-targeted gelatin nanoparticles for systemic administration of gemcitabine in an orthotopic pancreatic cancer model. Nanomed. Nanotechnol. Biol. Med. 12(3), 589-600 (2016)

159. Danesh, N.M., Lavaee, P., Ramezani, M., Abnous, K., Taghdisi, S.M.: Targeted and controlled release delivery of daunorubicin to T-cell acute lymphoblastic leukemia by aptamer-modified gold nanoparticles. Int. J. Pharm. 489(1), 311-317 (2015)

160. Chittasupho, C., Lirdprapamongkol, K., Kewsuwan, P., Sarisuta, N.: Targeted delivery of doxorubicin to A549 lung cancer cells by CXCR4 antagonist conjugated PLGA nanoparticles. Eur. J. Pharm. Biopharm. 88(2), 529-538 (2014)

161. Robinson, M., Lee, B.Y., Leonenko, Z.: Drugs and drug delivery systems targeting amyloid- $\beta$ in Alzheimer's. AIMS Mol. Sci. 2(3), 332-358 (2015)

162. Pahnke, J., Wolkenhauer, O., Krohn, M., Walker, L.C.: Clinicopathologic function of cerebral $\mathrm{ABC}$ transporters implications for the pathogenesis of Alzheimer's disease. Curr. Alzheimer Res. 5(4), 396 (2008)

163. Dinda, S.C., Pattnaik, G.: Nanobiotechnology-based drug delivery in brain targeting. Curr. Pharm. Biotechnol. 14(15), 1264-1274 (2013)

164. Kwon, H.J., Cha, M.-Y., Kim, D., Kim, D.K., Soh, M., Shin, K., Hyeon, T., Mook-Jung, I.: Mitochondria-targeting ceria nanoparticles as antioxidants for Alzheimer's disease. ACS Nano 10(2), 2860-2870 (2016)

165. Matveyenko, A.V., Butler, P.C.: Relationship between $\beta$-cell mass and diabetes onset. Diabetes Obes. Metab. 10(s4), 23-31 (2008)

166. Gendelman, H.E., Anantharam, V., Bronich, T., Ghaisas, S., Jin, H., Kanthasamy, A.G., Liu, X., McMillan, J., Mosley, R.L., Narasimhan, B.: Nanoneuromedicines for degenerative, inflammatory, and infectious nervous system diseases. Nanomed. Nanotechnol. Biol. Med. 11(3), 751-767 (2015)

167. Lu, H., Dai, Y., Lv, L., Zhao, H.: Chitosan-graft-polyethyleneimine/DNA nanoparticles as novel non-viral gene delivery vectors targeting osteoarthritis. PLoS ONE 9(1), e84703 (2014)

168. McDonagh, B.H.: Multifunctional nanoparticles for bioimaging (2015)

169. Qian, B.-Z., Pollard, J.W.: Macrophage diversity enhances tumor progression and metastasis. Cell 141(1), 39-51 (2010)

170. Rocha, V.Z., Libby, P.: Obesity, inflammation, and atherosclerosis. Nature Rev. Cardiol. 6(6), 399-409 (2009)

171. De Luca, C., Olefsky, J.M.: Inflammation and insulin resistance. FEBS Lett. 582(1), 97-105 (2008)

172. Lameijer, M.A., Tang, J., Nahrendorf, M., Beelen, R.H.J., Mulder, W.J.M.: Monocytes and macrophages as nanomedicinal targets for improved diagnosis and treatment of disease. Expert Rev. Mol. Diagn. 13(6), 567-580 (2013)

173. Moghimi, S.M., Parhamifar, L., Ahmadvand, D., Wibroe, P.P., Andresen, T.L., Farhangrazi, Z.S., Hunter, A.C.: Particulate systems for targeting of macrophages: basic and therapeutic concepts. J. Innate Immun. 4(5-6), 509-528 (2012)

174. Chellat, F., Merhi, Y., Moreau, A., Yahia, L.H.: Therapeutic potential of nanoparticulate systems for macrophage targeting. Biomaterials 26(35), 7260-7275 (2005)

175. Shilakari Asthana, G., Asthana, A., Kohli, D.V., Vyas, S.P.: Mannosylated chitosan nanoparticles for delivery of antisense oligonucleotides for macrophage targeting. BioMed Res. Int. 2014 (2014)

176. Gao, J., Chen, P., Singh, Y., Zhang, X., Szekely, Z., Stein, S., Sinko, P.J.: Novel monodisperse PEGtide dendrons: design, fabrication, and evaluation of mannose receptor-mediated macrophage targeting. Bioconjug. Chem. 24(8), 1332-1344 (2013)

177. Yu, S.S., Lau, C.M., Barham, W.J., Onishko, H.M., Nelson, C.E., Li, H., Smith, C.A., Yull, F.E., Duvall, C.L., Giorgio, T.D.: Macrophage-specific RNA interference targeting via click, mannosylated polymeric micelles. Mol. Pharm. 10(3), 975-987 (2013) 
178. Zhu, S., Niu, M., O'Mary, H., Cui, Z.: Targeting of tumorassociated macrophages made possible by PEG-sheddable, mannose-modified nanoparticles. Mol. Pharm. 10(9), 3525-3530 (2013)

179. Pruthi, J., Mehra, N.K., Jain, N.K.: Macrophages targeting of amphotericin B through mannosylated multiwalled carbon nanotubes. J. Drug Target. 20(7), 593-604 (2012)

180. Tiwari, S., Chaturvedi, A.P., Tripathi, Y.B., Mishra, B.: Macrophage-specific targeting of isoniazid through mannosylated gelatin microspheres. AAPS Pharm. Sci. Tech. 12(3), 900-908 (2011)

181. Wood, T.R., Chow, R.Y., Hanes, C.M., Zhang, X., Kashiwagi, K., Shirai, Y., Trebak, M., Loegering, D.J., Saito, N., Lennartz, M.R.: PKC-pseudosubstrate and catalytic activity are necessary for membrane delivery during IgG-mediated phagocytosis. J. Leukoc. Biol. 94(1), 109-122 (2013)

182. Zhao, C., Fan, T., Yang, Y., Wu, M., Li, L., Zhou, Z., Jian, Y., Zhang, Q., Huang, Y.: Preparation, macrophages targeting delivery and anti-inflammatory study of pentapeptide grafted nanostructured lipid carriers. Int. J. Pharm. 450(1), 11-20 (2013)

183. Jain, S., Amiji, M.: Tuftsin-modified alginate nanoparticles as a noncondensing macrophage-targeted DNA delivery system. Biomacromol 13(4), 1074-1085 (2012)

184. Betageri, G.V., Black, C.D.V., Szebeni, J., Wahl, L.M., Weinstein, J.N.: Fc-receptor-mediated targeting of antibody-bearing liposomes containing dideoxycytidine triphosphate to human monocyte/Macrophages. J. Pharm. Pharmacol. 45(1), 48-53 (1993)

185. Gu, X., Zhang, W., Liu, J., Shaw, J.P., Shen, Y., Xu, Y., Lu, H., $\mathrm{Wu}, \mathrm{Z}$.: Preparation and characterization of a lovastatin-loaded protein-free nanostructured lipid carrier resembling high-density lipoprotein and evaluation of its targeting to foam cells. AAPS Pharm. Sci. Tech. 12(4), 1200-1208 (2011)

186. Richman, M., Perelman, A., Gertler, A., Rahimipour, S.: Effective targeting of $A \beta$ to macrophages by sonochemically prepared surface-modified protein microspheres. Biomacromol 14(1), 110-116 (2013)

187. Kamat, M., El-Boubbou, K., Zhu, D.C., Lansdell, T., Lu, X., Li, W., Huang, X.: Hyaluronic acid immobilized magnetic nanoparticles for active targeting and imaging of macrophages. Bioconjug. Chem. 21(11), 2128-2135 (2010)

188. Gaspar, R.r., Prat, V., Opperdoes, F.R., Roland, M.: Macrophage activation by polymeric nanoparticles of polyalkylcyanoacrylates: activity against intracellular Leishmania donovani associated with hydrogen peroxide production. Pharm. Res. 9(6), 782-787 (1992)

189. Aouadi, M., Tesz, G.J., Nicoloro, S.M., Wang, M., Soto, E., Ostroff, G.R., Czech, M.P.: Orally delivered siRNA targeting macrophage Map4k4 suppresses systemic inflammation. Nature 458(7242), 1180-1184 (2009)

190. Huang, H., Ostroff, G.R., Lee, C.K., Agarwal, S., Ram, S., Rice, P.A., Specht, C.A., Levitz, S.M.: Relative contributions of dectin-1 and complement to immune responses to particulate $\beta$ glucans. J. Immunol. 189(1), 312-317 (2012)

191. Liby, K.T., Sporn, M.B.: Synthetic oleanane triterpenoids: multifunctional drugs with a broad range of applications for prevention and treatment of chronic disease. Pharmacol. Rev. 64(4), 972-1003 (2012)

192. Chen, X., Wong, R., Khalidov, I., Wang, A.Y., Leelawattanachai, J., Wang, Y., Jin, M.M.: Inflamed leukocyte-mimetic nanoparticles for molecular imaging of inflammation. Biomaterials 32(30), 7651-7661 (2011)

193. Sipkins, D.A., Gijbels, K., Tropper, F.O.D., Bednarski, M., Li, K.C.P., Steinman, L.: ICAM-1 expression in autoimmune encephalitis visualized using magnetic resonance imaging. J. Neuroimmunol. 104(1), 1-9 (2000)
194. Weller, G.E.R., Lu, E., Csikari, M.M., Klibanov, A.L., Fischer, D., Wagner, W.R., Villanueva, F.S.: Ultrasound imaging of acute cardiac transplant rejection with microbubbles targeted to intercellular adhesion molecule-1. Circulation 108(2), 218-224 (2003)

195. Zhang, N., Chittasupho, C., Duangrat, C., Siahaan, T.J., Berkland, C.: PLGA nanoparticle peptide conjugate effectively targets intercellular cell-adhesion molecule-1. Bioconjug. Chem. 19(1), 145-152 (2007)

196. Kelly, K.A., Allport, J.R., Tsourkas, A., Shinde-Patil, V.R., Josephson, L., Weissleder, R.: Detection of vascular adhesion molecule-1 expression using a novel multimodal nanoparticle. Circ. Res. 96(3), 327-336 (2005)

197. Voinea, M., Manduteanu, I., Dragomir, E., Capraru, M., Simionescu, M.: Immunoliposomes directed toward VCAM-1 interact specifically with activated endothelial cells-a potential tool for specific drug delivery. Pharm. Res. 22(11), 1906-1917 (2005)

198. Nahrendorf, M., Keliher, E., Panizzi, P., Zhang, H., Hembrador, S., Figueiredo, J.-L., Aikawa, E., Kelly, K., Libby, P., Weissleder, R.: 18F-4 V for PET CT Imaging of VCAM-1 Expression in Atherosclerosis. JACC: Cardiov. Imaging 2(10), 1213-1222 (2009)

199. Chan, J.M., Zhang, L., Tong, R., Ghosh, D., Gao, W., Liao, G., Yuet, K.P., Gray, D., Rhee, J.-W., Cheng, J.: Spatiotemporal controlled delivery of nanoparticles to injured vasculature. Proc. Natl. Acad. Sci. 107(5), 2213-2218 (2010)

200. Ehrhardt, C., Kneuer, C., Bakowsky, U.: Selectin-an emerging target for drug delivery. Adv. Drug Deliv. Rev. 56(4), 527-549 (2004)

201. Dustin, M.L., Rothlein, R., Bhan, A.K., Dinarello, C.A., Springer, T.A.: Induction by IL 1 and interferon-gamma: tissue distribution, biochemistry, and function of a natural adherence molecule (ICAM-1). J. Immunol. 137(1), 245-254 (1986)

202. Kang, S., Park, T., Chen, X., Dickens, G., Lee, B., Lu, K., Rakhilin, N., Daniel, S., Jin, M.M.: Tunable physiologic interactions of adhesion molecules for inflamed cell-selective drug delivery. Biomaterials 32(13), 3487-3498 (2011)

203. Gottesman, M.M., Fojo, T., Bates, S.E.: Multidrug resistance in cancer: role of ATP-dependent transporters. Nat. Rev. Cancer 2(1), 48-58 (2002)

204. Thomas, H., Coley, H.M.: Overcoming multidrug resistance in cancer: an update on the clinical strategy of inhibiting p-glycoprotein. Cancer Control 10(2), 159 (2003)

205. Choi, C.-H.: ABC transporters as multidrug resistance mechanisms and the development of chemosensitizers for their reversal. Cancer Cell Int. 5(1), 1 (2005)

206. Gillet, J.-P., Gottesman, M.M.: Mechanisms of multidrug resistance in cancer. Multi-drug Resistance in Cancer. Methods Mol. Biol. 47-76 (2010)

207. Jain, R.K.: Transport of molecules in the tumor interstitium: a review. Can. Res. 47(12), 3039-3051 (1987)

208. Zahreddine, H., Borden, K.L.: Mechanisms and insights into drug resistance in cancer. Frontiers in Pharmacology 4(28.10), 3389 (2013)

209. Sampath, D., Cortes, J., Estrov, Z., Du, M., Shi, Z., Andreeff, M., Gandhi, V., Plunkett, W.: Pharmacodynamics of cytarabine alone and in combination with 7-hydroxystaurosporine (UCN01) in AML blasts in vitro and during a clinical trial. Blood 107(6), 2517-2524 (2006)

210. Michael, M., Doherty, M.M.: Tumoral drug metabolism: overview and its implications for cancer therapy. J. Clin. Oncol. 23(1), 205-229 (2005)

211. Demant, E.J.F., Sehested, M., Jensen, P.B.: A model for computer simulation of P-glycoprotein and transmembrane $\hat{\mathrm{I}}$ " $\mathrm{pH}$ mediated anthracycline transport in multidrug-resistant tumor 
cells. Biochimica et Biophysica Acta (BBA)-Molecular. Cell Res. 1055(2), 117-125 (1990)

212. Ullah, M.F.: Cancer multidrug resistance (MDR): a major impediment to effective chemotherapy. Asian Pac. J. Cancer Prev. 9(1), 1-6 (2008)

213. Gottesman, M.M.: Mechanisms of cancer drug resistance. Annu. Rev. Med. 53(1), 615-627 (2002)

214. Leonard, G.D., Fojo, T., Bates, S.E.: The role of ABC transporters in clinical practice. Oncologist 8(5), 411-424 (2003)

215. Liu, F.-S.: Mechanisms of chemotherapeutic drug resistance in cancer therapy: a quick review. Taiwanese J. Obstet. Gynecol. 48(3), 239-244 (2009). doi:10.1016/S1028-4559 (09)60296-5

216. van de Ven, R., Oerlemans, R., van der Heijden, J.W., Scheffer, G.L., de Gruijl, T.D., Jansen, G., Scheper, R.J.: ABC drug transporters and immunity: novel therapeutic targets in autoimmunity and cancer. J. Leukoc. Biol. 86(5), 1075-1087 (2009)

217. Riordan, J.R., Deuchars, K., Kartner, N., Alon, N., Trent, J., Ling, V.: Amplification of P-glycoprotein genes in multidrugresistant mammalian cell lines. Nature 316(6031), 817-819 (1985)

218. Doyle, L.A., Yang, W., Abruzzo, L.V., Krogmann, T., Gao, Y., Rishi, A.K., Ross, D.D.: A multidrug resistance transporter from human MCF-7 breast cancer cells. Proc. Natl. Acad. Sci. 95(26), 15665-15670 (1998)

219. Borst, P., Evers, R., Kool, M., Wijnholds, J.: A family of drug transporters: the multidrug resistance-associated proteins. J. Natl Cancer Inst. 92(16), 1295-1302 (2000)

220. Juliano, R.L., Ling, V.: A surface glycoprotein modulating drug permeability in Chinese hamster ovary cell mutants. Biochimica et Biophysica Acta (BBA)-Biomembranes 455(1), 152-162 (1976)

221. Ambudkar, S.V., Dey, S., Hrycyna, C.A., Ramachandra, M., Pastan, I., Gottesman, M.M.: Biochemical, cellular, and pharmacological aspects of the multidrug transporter 1. Ann. Rev. Pharmacol. Toxicol. 39(1), 361-398 (1999)

222. Dean, M., Fojo, T., Bates, S.: Tumour stem cells and drug resistance. Nat. Rev. Cancer 5(4), 275-284 (2005)

223. Vinogradov, S., Wei, X.: Cancer stem cells and drug resistance: the potential of nanomedicine. Nanomedicine 7(4), 597-615 (2012)

224. Charafe-Jauffret, E., Monville, F., Ginestier, C., Dontu, G., Birnbaum, D., Wicha, M.S.: Cancer stem cells in breast: current opinion and future challenges. Pathobiology 75(2), 75-84 (2008)

225. Ponti, D., Costa, A., Zaffaroni, N., Pratesi, G., Petrangolini, G., Coradini, D., Pilotti, S., Pierotti, M.A., Daidone, M.G.: Isolation and in vitro propagation of tumorigenic breast cancer cells with stem/progenitor cell properties. Can. Res. 65(13), 5506-5511 (2005)

226. Prince, M.E., Sivanandan, R., Kaczorowski, A., Wolf, G.T., Kaplan, M.J., Dalerba, P., Weissman, I.L., Clarke, M.F., Ailles, L.E.: Identification of a subpopulation of cells with cancer stem cell properties in head and neck squamous cell carcinoma. Proc. Natl. Acad. Sci. 104(3), 973-978 (2007)

227. Clarke, M.F., Dick, J.E., Dirks, P.B., Eaves, C.J., Jamieson, C.H.M., Jones, D.L., Visvader, J., Weissman, I.L., Wahl, G.M.: Cancer stem cells-perspectives on current status and future directions: AACR Workshop on cancer stem cells. Can. Res. 66(19), 9339-9344 (2006)

228. Dick, D.: Human acute myeloid leukemia is organized as a hierarchy that originates from a primitive hematopoietic cell. Nat. Med. 3, 730-737 (1997)

229. Hotamisligil, G.k.S.: Inflammation and metabolic disorders. Nature 444(7121), 860-867 (2006)
230. Facchini, F.S., Hua, N., Abbasi, F., Reaven, G.M.: Insulin resistance as a predictor of age-related diseases. J. Clin. Endocrinol. Metab. 86(8), 3574-3578 (2001)

231. Loscher, W., Potschka, H.: Drug resistance in brain diseases and the role of drug efflux transporters. Nat. Rev. Neurosci. 6(8), 591-602 (2005)

232. Doan, K.M.M., Humphreys, J.E., Webster, L.O., Wring, S.A., Shampine, L.J., Serabjit-Singh, C.J., Adkison, K.K., Polli, J.W.: Passive permeability and P-glycoprotein-mediated efflux differentiate central nervous system (CNS) and non-CNS marketed drugs. J. Pharmacol. Exp. Ther. 303(3), 1029-1037 (2002)

233. Bartok, B., Firestein, G.S.: Fibroblast-like synoviocytes: key effector cells in rheumatoid arthritis. Immunol. Rev. 233(1), 233-255 (2010)

234. Morgan, C., Lunt, M., Brightwell, H., Bradburn, P., Fallow, W., Lay, M., Silman, A., Bruce, I.N.: Contribution of patient related differences to multidrug resistance in rheumatoid arthritis. Ann. Rheum. Dis. 62(1), 15-19 (2003)

235. Sharma, G., Sharma, A.R., Nam, J.-S., Doss, G.P.C., Lee, S.-S., Chakraborty, C.: Nanoparticle based insulin delivery system: the next generation efficient therapy for Type 1 diabetes. J. Nanobiotechnol. 13(1), 1 (2015)

236. Shaji, J., Patole, V.: Protein and peptide drug delivery: oral approaches. Indian J. Pharm. Sci. (2008)

237. Bhumkar, D.R., Joshi, H.M., Sastry, M., Pokharkar, V.B.: Chitosan reduced gold nanoparticles as novel carriers for transmucosal delivery of insulin. Pharm. Res. 24(8), 1415-1426 (2007)

238. Kudva, Y.C., Carter, R.E., Cobelli, C., Basu, R., Basu, A.: Closed-loop artificial pancreas systems: physiological input to enhance next-generation devices. Diabetes Care 37(5), 1184-1190 (2014)

239. Zhang, F., Xu, C.-L., Liu, C.-M.: Drug delivery strategies to enhance the permeability of the blood-brain barrier for treatment of glioma. Drug Design Dev. Therapy 9, 2089-2100 (2015)

240. Brem, H., Mahaley Jr., M.S., Vick, N.A., Black, K.L., Schold Jr., S.C., Burger, P.C., Friedman, A.H., Ciric, I.S., Eller, T.W., Cozzens, J.W.: Interstitial chemotherapy with drug polymer implants for the treatment of recurrent gliomas. J. Neurosurg. 74(3), 441-446 (1991)

241. Grossman, S.A., Reinhard, C., Colvin, O.M., Chasin, M., Brundrett, R., Tamargo, R.J., Brem, H.: The intracerebral distribution of BCNU delivered by surgically implanted biodegradable polymers. J. Neurosurg. 76(4), 640-647 (1992)

242. Ellington, A.D., Szostak, J.W.: In vitro selection of RNA molecules that bind specific ligands. Nature 346(6287), 818-822 (1990)

243. Tuerk, C., Gold, L.: Systematic evolution of ligands by exponential enrichment: RNA ligands to bacteriophage T4 DNA polymerase. Science 249(4968), 505-510 (1990)

244. Schneider, D.J., Feigon, J., Hostomsky, Z., Gold, L.: Highaffinity ssDNA inhibitors of the reverse transcriptase of type 1 human immunodeficiency virus. Biochemistry 34(29), 9599-9610 (1995)

245. Mosing, R.K., Mendonsa, S.D., Bowser, M.T.: Capillary electrophoresis-SELEX selection of aptamers with affinity for HIV1 reverse transcriptase. Anal. Chem. 77(19), 6107-6112 (2005)

246. Chen, F., Hu, Y., Li, D., Chen, H., Zhang, X.-L.: CS-SELEX generates high-affinity ssDNA aptamers as molecular probes for hepatitis C virus envelope glycoprotein E2. PLoS ONE 4(12), e8142 (2009)

247. Ng, E.W.M., Shima, D.T., Calias, P., Cunningham, E.T., Guyer, D.R., Adamis, A.P.: Pegaptanib, a targeted anti-VEGF aptamer for ocular vascular disease. Nat. Rev. Drug Discovery 5(2), 123-132 (2006)

248. Farokhzad, O.C., Jon, S., Khademhosseini, A., Tran, T.-N.T., LaVan, D.A., Langer, R.: Nanoparticle-aptamer bioconjugates a 
new approach for targeting prostate cancer cells. Can. Res. 64(21), 7668-7672 (2004)

249. Cerchia, L., Giangrande, P.H., McNamara, J.O., de Franciscis, V.: Cell-specific aptamers for targeted therapies. Nucleic Acid Peptide Aptamers Methods Protocols, 59-78 (2009)

250. Cerchia, L., de Franciscis, V.: Targeting cancer cells with nucleic acid aptamers. Trends Biotechnol. 28(10), 517-525 (2010)

251. Sefah, K., Shangguan, D., Xiong, X., O’Donoghue, M.B., Tan, W.: Development of DNA aptamers using Cell-SELEX. Nat. Protoc. 5(6), 1169-1185 (2010)

252. Meng, H., Liong, M., Xia, T., Li, Z., Ji, Z., Zink, J.I., Nel, A.E.: Engineered design of mesoporous silica nanoparticles to deliver doxorubicin and P-glycoprotein siRNA to overcome drug resistance in a cancer cell line. ACS Nano 4(8), 4539-4550 (2010)

253. Zhao, J., Feng, S.-S.: Nanocarriers for delivery of siRNA and co-delivery of siRNA and other therapeutic agents. Nanomedicine 10(14), 2199-2228 (2015)

254. Saul, J.M., Annapragada, A.V., Bellamkonda, R.V.: A dual-ligand approach for enhancing targeting selectivity of therapeutic nanocarriers. J. Control. Release 114(3), 277-287 (2006)
255. Reardon, D.A., Nabors, L.B., Stupp, R., Mikkelsen, T.: Cilengitide: an integrin-targeting arginine-glycine-aspartic acid peptide with promising activity for glioblastoma multiforme. Expert Opin. Investig. Drugs 17(8), 1225-1235 (2008)

256. Duskey, J.T., Rice, K.G.: Nanoparticle ligand presentation for targeting solid tumors. AAPS Pharm. Sci. Tech. 15(5), 1345-1354 (2014)

257. Gao, Z., Zhang, L., Sun, Y.: Nanotechnology applied to overcome tumor drug resistance. J. Control. Release 162(1), 45-55 (2012)

258. Chen, H.-H., Huang, W.-C., Chiang, W.-H., Liu, T.-I., Shen, M.Y., Hsu, Y.-H., Lin, S.-C., Chiu, H.-C.: pH-Responsive therapeutic solid lipid nanoparticles for reducing P-glycoproteinmediated drug efflux of multidrug resistant cancer cells. Int. J. Nanomed. 10, 5035 (2015)

259. Rao, W., Wang, H., Han, J., Zhao, S., Dumbleton, J., Agarwal, P., Zhang, W., Zhao, G., Yu, J., Zynger, D.L.: Chitosan-decorated doxorubicin-encapsulated nanoparticle targets and eliminates tumor reinitiating cancer stem-like cells. ACS Nano 9(6), 5725-5740 (2015)

260. Hu, Y., Fu, L.: Targeting cancer stem cells: a new therapy to cure cancer patients. Am. J. Cancer Res. 2(3), 340-356 (2012) 